\title{
A Systematic Review of Organic Versus Conventional Food Consumption: Is There a Measurable Benefit on Human Health?
}

\author{
Vanessa Vigar 1,2,3,4, Stephen Myers ${ }^{1,3,4, * \mathbb{C}}$, Christopher Oliver 1,3,4,5® , Jacinta Arellano ${ }^{3}$, \\ Shelley Robinson ${ }^{1,3,4}$ and Carlo Leifert ${ }^{4}$ (D) \\ 1 NatMed Research, Southern Cross University, Lismore NSW 2480, Australia; \\ vanessa.vigar@scu.edu.au (V.V.); christopher.oliver@scu.edu.au (C.O.); shelley.robinson@scu.edu.au (S.R.) \\ 2 Integria Healthcare, Eight Mile Plains QLD 4113, Australia \\ 3 School of Health and Human Sciences, Southern Cross University, Lismore NSW 2480, Australia; \\ jacinta.arellano@scu.edu.au \\ 4 Centre for Organics Research, Southern Cross University, Lismore NSW 2480, Australia; \\ carlo.leifert@scu.edu.au \\ 5 Oliver Nutrition Pty Ltd, Lismore NSW 2480, Australia \\ * Correspondence: stephen.myers@scu.edu.au; Tel.: +61-421612713
}

Received: 25 September 2019; Accepted: 13 December 2019; Published: 18 December 2019

\begin{abstract}
The current review aims to systematically assess the evidence related to human health outcomes when an organic diet is consumed in comparison to its conventional counterpart. Relevant databases were searched for articles published to January 2019. Clinical trials and observational research studies were included where they provided comparative results on direct or indirect health outcomes. Thirty-five papers met the criteria for inclusion in the review. Few clinical trials assessed direct improvements in health outcomes associated with organic food consumption; most assessed either differences in pesticide exposure or other indirect measures. Significant positive outcomes were seen in longitudinal studies where increased organic intake was associated with reduced incidence of infertility, birth defects, allergic sensitisation, otitis media, pre-eclampsia, metabolic syndrome, high BMI, and non-Hodgkin lymphoma. The current evidence base does not allow a definitive statement on the health benefits of organic dietary intake. However, a growing number of important findings are being reported from observational research linking demonstrable health benefits with organic food consumption. Future clinical research should focus on using long-term whole-diet substitution with certified organic interventions as this approach is more likely to determine whether or not true measurable health benefits exist.
\end{abstract}

Keywords: health outcomes; organic; organic diet; pesticide-free; sustainable diet

\section{Introduction}

The global marketplace of organics has grown rapidly over the last few decades and consumer demand for organic products is increasing globally, with approximately 80 billion Euros ( $\$ 92$ billion USD) spent on organic products annually [1]. A recent report from the Research Institute of Organic Agriculture (FiBL) and IFOAM Organics International, shows a 14.7\% increase in organic farmland from 2014 to 2015, totalling 50.9 million hectares, with Australia having the largest amount of agricultural land at 22.7 million hectares [2]. Organic food items most often consumed in Europe are organic baby foods followed by organic eggs, fruit and vegetables, then dairy products, with organic dairy reaching market shares of around 10 percent of overall sales in some European countries [2]. In the United States, fruit and vegetables make up the largest areas of organic food sales, followed by dairy products [3]. 
The reasons consumers are increasingly choosing organic over conventional food products are varied, including many reasons beside personal health and wellbeing, such as environmental concerns or animal welfare impact. However, the major determinants behind consumer purchase of organic products, is the belief that organic food is healthier or has a superior nutritional profile [4-6].

Regular consumers of organic food are most likely to be female, health-conscious, physically active, and in the higher brackets of education and income than their non-organic consuming counterparts $[7,8]$. They are also more likely to have a higher ratio of plant to animal foods, with a strong relationship between vegetarian/vegan consumers and organic consumption [7,9]. This consumer group generally has an increased wholefood dietary intake, as a result of both the general ethos of organic consumers (i.e., preference over processed/ultra-processed foods), and restricted use of additives in organic processed foods. Diet composition between organic and non-organic consumers may, therefore, be quite different.

The notion that organic food may be healthier has some support. Although there appears to be little variation between organic and conventional food products in terms of macro nutritional value (protein, fat, carbohydrate and dietary fibre), other compositional differences have been demonstrated. These include higher antioxidant concentrations (particularly polyphenols) in organic crops [10]; increased levels of omega-3 fatty acids in organic dairy products [11-13]; and improved fatty acid profiles in organic meat products $[14,15]$. These compositional differences are comprehensively discussed in several recent reviews [16-19]. There is preliminary evidence to suggest that these compositional differences may have an effect on plasma levels of certain nutrients including magnesium, fat-soluble micronutrients ( $\alpha$-carotene, $\beta$-carotene, lutein, and zeaxanthin), and fatty acids (linoleic, palmitoleic, $\gamma$-linolenic, and docosapentaenoic acids) [20]. Any possible clinical effects of such differences need further investigation.

Likely to be of more importance than compositional differences between the two, is what organic foods do not contain. Organic foods have been shown to have lower levels of toxic metabolites, including heavy metals such as cadmium, and synthetic fertilizer and pesticide residues [10,17]. Consumption of organic foods may also reduce exposure to antibiotic-resistant bacteria [19].

The long-term safety of pesticide consumption through conventional food production has been questioned, with evidence from long-term cohort studies covering areas ranging from possible neurotoxicity to endocrine disruption [21]. A number of widely used pesticides have been banned retrospectively only when unexpected negative health impacts have been identified [22,23]. From a regulatory perspective, dietary intake of pesticides is not considered to pose a health risk to consumers as long as individual pesticide concentrations in foods are below the Maximum Residue Level (MRL). Surveys conducted by both the European Food Safety Authority and the United States Department of Agriculture show that the vast majority of foods contained individual pesticide levels below the MRL, at $1.7 \%$ and $0.59 \%$, respectively, found to exceed the limits. It was also found that $30.1 \%$ and $27.5 \%$, respectively, of food samples analysed contained multiple pesticide residues $[24,25]$. One of the main criticisms of current regulatory pesticide approval processes is that they do not require safety testing of pesticide mixtures or formulations of pesticides $[23,26,27]$. There is considerable controversy about health risks posed by chronic low-level dietary pesticide exposure [28-30], and whilst lower levels of pesticide residue excretion is consistently observed during organic diet intakes [31-34], there is uncertainty around how this may impact the health of the consumer.

The last systematic review into the effect of organic food consumption on health was conducted by Dangour et al. in 2010 [35], which was limited to strict inclusion criteria of organic interventions, and Smith-Spangler et al. in 2012 [19], which contained only minimal focus on the human health effects of organic food, and a broader focus on nutritional content of organically and conventionally grown food and food safety. Although there have been other more recent reviews on the effects of organic diet on broader aspects of health [16-18,21], none have been systematic. The literature has expanded since these earlier systematic reviews, with many cohort and cross-sectional studies being published which compare organic versus conventional dietary intake on a range of health outcomes. 
Dangour et al. (2010) included 12 reports overall, of which eight were human studies (six clinical trials, one cohort study, 1 cross-section study), and four that reported animal or in vitro research. The Smith-Spangler et al. (2012) report was more comprehensive, including 17 human studies (in addition to 223 studies of comparative nutrient/contaminant profiles).

The present systematic review was designed to assess the breadth of evidence related to human health outcomes when an organic diet is consumed in comparison to its conventional counterpart. This review reports results from 35 studies including both clinical trial and observational research and includes substantially more papers than previous systematic reviews on this topic. This review does not include a comparison of nutritional quality between production types, safety of organic food, or human studies where environmental pesticide exposure is the focus.

\section{Methods}

\subsection{Literature Search}

This systematic review has been conducted in accordance with the guidelines of the Preferred Reporting Items of Systematic Reviews and Meta-analysis (PRISMA) statement [36].

Relevant studies were identified by a systematic search from the Cochrane, MEDLINE, EMBASE, and TOXNET databases for articles published in January 2019. Relevant keywords included terms related to organic dietary intake in combination with words relevant to health outcomes (i.e., asthma, eczema, obesity, diabetes). Search terms were amended slightly for each database. Articles with English titles and abstracts were considered for inclusion. The search strategy was developed by two authors (SM and VV) and was performed by VV in January 2019. Additional publications were identified from the reference lists of obtained articles that were included in the review. Refer to Supplementary Figure S1 (see online Supplementary Material).

All articles that compared organic versus conventional dietary intake in relation to a direct or an indirect health outcome were included. We did not set out to limit paper inclusion by including a strict definition of organic intake, but accepted all papers that self-identified as representing comparative information on health outcomes from organic versus conventional diets. In doing so, we set out a priori to ensure we obtained a comprehensive snapshot of the available literature in this area.

\subsection{Study Eligibility Criteria}

\subsubsection{Population}

Only human feeding studies were included. Studies including infant participants measured from the second trimester of pregnancy were included where the mother gave dietary information during pregnancy.

\subsubsection{Intervention}

Any clinical trial where organic food items were taken to replace non-organic food items, or observational studies where there was a comparison between organic and non-organic dietary intake were included. This encompassed individual food or drink replacement, through to entire diet substitution. Observational research was accepted where dietary intake was classified according to level of organic food within individual dietary groups or whole diet.

\subsubsection{Outcome}

Clinical trials were included where they provided comparative results on direct or indirect health outcomes. Cohort studies were included where associations with development of disorder or disease were reported, or if they provided comparisons of biological samples across organic versus conventional dietary intake groups. 


\subsubsection{Study Designs}

Types of studies included were randomised controlled trials (RCT), non-controlled trials, prospective or retrospective cohort studies, case-control studies and cross-sectional studies.

\subsubsection{Exclusion Criteria}

Articles were excluded if they were not specifically examining the effect of organic dietary intake with conventional dietary intake, or if they did not report on human biomarkers related to health, or disease development. Articles were excluded if they were concerned with occupational exposure to agricultural chemicals or domestic use of pesticides and unrelated to dietary consumption of organic versus non-organic foods.

\subsection{Data Extraction}

Two reviewers independently reviewed full articles for inclusion based on relevance to the study question and eligibility criteria. One reviewer (VV) extracted data from included studies, which was checked by a separate reviewer (SM). The details are presented in Tables 1 and 2, using the following parameters: (i) author and year of publication; (ii) study population including country of origin and key demographic detail; (iii) sample size; (iv) study design and duration of intervention/exposure; (vi) exposure to organic diet and comparator; (vii) outcomes assessed; (viii) results; (ix) organic definition. 
Table 1. Data extraction table-Clinical trials.

\begin{tabular}{|c|c|c|c|c|c|c|c|}
\hline Ref. & Study population & $n$ & $\begin{array}{l}\text { Design and } \\
\text { Duration }\end{array}$ & Exposure/treatment & Outcome measures & Results & Definition of organic \\
\hline $\begin{array}{c}\text { Caris-Veyrant } \\
(2004) \\
{[37]}\end{array}$ & $\begin{array}{c}\text { France } \\
\text { Age: } 21-39 \text { years } \\
\text { 100\% female } \\
\text { Healthy }\end{array}$ & 24 & $\begin{array}{c}\text { Parallel RCT. } \\
\text { Single-blind. } \\
\text { 2-arm, 3 week } \\
\text { dietary } \\
\text { intervention. }\end{array}$ & $\begin{array}{l}100 \mathrm{~g} \text { of conventional } \\
\text { tomato puree (NO), or } \\
\text { organic tomato puree } \\
\text { (O) added to lunch or } \\
\text { dinner once daily. }\end{array}$ & $\begin{array}{c}\text { Plasma Vit } C, \beta \text {-carotene and } \\
\text { lycopene. }\end{array}$ & $\begin{array}{l}\text { Tomato puree increased plasma } \\
\beta \text {-carotene and lycopene in both } \\
\text { groups. There were no } \\
\text { significant between-group } \\
\text { differences in any outcome. The } \\
\text { two purees had similar } \\
\text { lycopene and beta-carotene } \\
\text { contents. }\end{array}$ & $\begin{array}{l}\text { Describes growing } \\
\text { conditions of both crops. } \\
\text { They were both } \\
\text { experimental crops for the } \\
\text { study. }\end{array}$ \\
\hline $\begin{array}{c}\text { Stracke (2009) } \\
{[38]}\end{array}$ & $\begin{array}{c}\text { Germany } \\
\text { Age: } 19-54 \text { years } \\
100 \% \text { male } \\
\text { Healthy }\end{array}$ & 36 & $\begin{array}{l}\text { Parallel RCT. } \\
\text { Double-blind. } \\
\text { 3-arm, 14 day } \\
\text { intervention, with } \\
\text { 4-week low } \\
\text { carotenoid diet } \\
\text { prior to baseline. }\end{array}$ & $\begin{array}{c}200 \mathrm{~g} \text { of conventional } \\
\text { blanched carrots (NO) } \\
\text { or organic blanched } \\
\text { carrots }(\mathrm{O}) \text { consumed } \\
\text { with main meal and } \\
\text { minimum } 10 \mathrm{~g} \text { fat. } \\
\text { Control (C) followed } \\
\text { carotenoid restricted } \\
\text { diet. }\end{array}$ & $\begin{array}{c}\text { Plasma carotenoid } \\
\text { concentration ( } \alpha \text { - and } \\
\beta \text {-carotene, lycopene, lutein, } \\
\text { zeaxanthin and } \\
\text { b-cryptoxanthin), Vit E, Vit C, } \\
\text { antioxidant activity (FRAP, } \\
\text { ORAC, TEAC), and LDL } \\
\text { oxidation; cytokine quantity, } \\
\text { NK cell quantity and activity, } \\
\text { DNA damage, plasma glucose, } \\
\text { uric acid, TAG, cholesterol. }\end{array}$ & $\begin{array}{l}\text { No significant difference in any } \\
\text { outcome measure. There was } \\
\text { no significant difference in } \\
\text { carotenoid concentration of } \mathrm{O} \\
\text { and NO carrots. }\end{array}$ & $\begin{array}{l}\text { The fertilisation, harvest } \\
\text { and distribution of the } \\
\text { carrots were monitored by } \\
\text { the Institute of Organic } \\
\text { Farming of the Johann } \\
\text { Heinrich von Thünen } \\
\text { Institute, Federal Research } \\
\text { Institute for Rural Areas, } \\
\text { Forestry and Fisheries at } \\
\text { Westerau. }\end{array}$ \\
\hline $\begin{array}{c}\text { Stracke (2010) a) } \\
\text { [39] }\end{array}$ & $\begin{array}{c}\text { Germany } \\
\text { Study 1: } \\
\text { Age: } 23-32 \text { years } \\
\text { 100\% male } \\
\text { Healthy }\end{array}$ & 6 & $\begin{array}{l}\text { Crossover. } \\
\text { Double-blind RCT. } \\
2 \text { phases, single } \\
\text { consumption after } \\
\text { overnight fast. }\end{array}$ & $\begin{array}{l}1000 \mathrm{~g} \text { of conventional } \\
\text { apples }(\mathrm{NO}) \text { or } \\
\text { organic apples }(\mathrm{O})\end{array}$ & $\begin{array}{l}\text { Apple polyphenols and their } \\
\text { metabolites, total antioxidant } \\
\text { status (FRAP, TEAC, and } \\
\text { ORAC). }\end{array}$ & $\begin{array}{l}\text { There were no significant } \\
\text { differences between the } \mathrm{O} \text { and } \\
\text { NO intake in any of the } \\
\text { analysed polyphenols. Apple } \\
\text { consumption had no effect on } \\
\text { TEAC, ORAC or FRAP. }\end{array}$ & $\begin{array}{l}\text { Cultivated according to the } \\
\text { requirements of "Bio Suisse" } \\
\text { (predominant label } \\
\text { organisation for certified } \\
\text { organic production in } \\
\text { Switzerland). }\end{array}$ \\
\hline $\begin{array}{c}\text { Stracke (2010) b) } \\
\text { [39] }\end{array}$ & $\begin{array}{l}\text { Germany } \\
\text { Study 2: } \\
\text { Age: (22-40yrs) } \\
\text { 100\% male } \\
\text { Healthy }\end{array}$ & 43 & $\begin{array}{c}\text { Parallel RCT } \\
\text { Double-blind. } \\
\text { 3-arm, 5-week } \\
\text { study (1-week } \\
\text { depletion period } \\
\text { /4-week } \\
\text { intervention). }\end{array}$ & $\begin{array}{l}500 \mathrm{~g} \text { of conventional } \\
\text { apples }(\mathrm{NO}) \text { or organic } \\
\text { apples }(\mathrm{O}) \text {. The third } \\
\text { group served as } \\
\text { control group (C), and } \\
\text { maintained an apple- } \\
\text { and polyphenol } \\
\text { restricted diet. }\end{array}$ & $\begin{array}{l}\text { Apple polyphenols and their } \\
\text { metabolites, glucose, TAG, } \\
\text { cholesterol, WBC, and uric acid; } \\
\text { total antioxidant status (FRAP, } \\
\text { TEAC, and ORAC); Vit C, Vit E, } \\
\text { carotenoids. }\end{array}$ & $\begin{array}{c}\text { No between group differences } \\
\text { between O and NO groups in } \\
\text { plasma glucose, uric acid, TAG, } \\
\text { cholesterol, Vit C, Vit E, } \\
\text { carotenoids, WBC, polyphenol } \\
\text { concentrations, or antioxidant } \\
\text { markers. }\end{array}$ & $\begin{array}{l}\text { Cultivated according to the } \\
\text { requirements of "Bio Suisse" } \\
\text { (predominant label } \\
\text { organisation for certified } \\
\text { organic production in } \\
\text { Switzerland). }\end{array}$ \\
\hline
\end{tabular}


Table 1. Cont

\begin{tabular}{|c|c|c|c|c|c|c|c|}
\hline Ref. & Study population & $n$ & $\begin{array}{l}\text { Design and } \\
\text { Duration }\end{array}$ & Exposure/treatment & Outcome measures & Results & Definition of organic \\
\hline $\begin{array}{c}\text { Briviba (2007) } \\
\text { [40] }\end{array}$ & $\begin{array}{c}\text { Germany } \\
\text { Age: } 23-32 \text { years } \\
100 \% \text { male } \\
\text { Healthy }\end{array}$ & 6 & $\begin{array}{c}\text { Crossover. } \\
\text { Double-blind RCT. } \\
2 \text { phases. 3-day } \\
\text { polyphenol } \\
\text { depletion prior to } \\
\text { single } \\
\text { consumption (after } \\
\text { overnight fast), } \\
\text { 1-week washout. }\end{array}$ & $\begin{array}{l}\text { Single consumption of } \\
1000 \mathrm{~g} \text { of conventional } \\
\text { apples (NO) or } \\
\text { organic apples }(\mathrm{O}) .\end{array}$ & $\begin{array}{c}\text { Antioxidant activity, LDL } \\
\text { oxidation, DNA damage (comet } \\
\text { assay). }\end{array}$ & $\begin{array}{l}\text { There were no statistically } \\
\text { significant differences between } \\
\text { groups on DNA damage, } \\
\text { antioxidant activity or LDL } \\
\text { oxidation. }\end{array}$ & $\begin{array}{l}\text { Cultivated according to the } \\
\text { requirements of "Bio Suisse' } \\
\text { (predominant label } \\
\text { organisation for certified } \\
\text { organic production in } \\
\text { Switzerland). }\end{array}$ \\
\hline $\begin{array}{c}\text { Grinder-Pedersen } \\
\text { (2003) } \\
{[41]}\end{array}$ & $\begin{array}{c}\text { Denmark } \\
\text { Age: } 21-35 \text { years } \\
6 \text { males }+10 \\
\text { females } \\
\text { Healthy }\end{array}$ & 16 & $\begin{array}{l}\text { Crossover. } \\
\text { Double-blind RCT. } \\
2 \text { phases. 1 week } \\
\text { run-in (excluding } \\
\text { flavonoid-containing } \\
\text { foods), } 22 \text { days } \\
\text { each intervention } \\
\text { with 3-week } \\
\text { washout. }\end{array}$ & $\begin{array}{l}\text { Whole diet } \\
\text { intervention. Two } \\
\text { intervention diets: } \\
\text { conventional (NO) } \\
\text { and organic (O); } \\
\text { consisted of } 4 \text { different } \\
\text { menus with identical } \\
\text { meals and quantities. }\end{array}$ & $\begin{array}{l}\text { SOD, Gpx, GR, Cat, TEAC, } \\
\text { FRAP, malondialdehyde, } \\
\text { 22-AAS. 24hr urine samples } \\
\text { (Days } 0 \text { and 22): measured } \\
\text { flavonoids (quercetin, } \\
\text { kaempferol, and isorhamnetin) } \\
\text { and flavonones (naringenin and } \\
\text { hesperitin). }\end{array}$ & $\begin{array}{l}\text { Quercetin }(p<0.01) \text { and 2-AAS } \\
(p<0.05) \text { were significantly } \\
\text { higher, as was urinary excretion } \\
\text { of quercetin and kaempferol }(p \\
<0.05) \text { in O phase. TEAC was } \\
\text { significantly increased }(p<0.05) \\
\text { after intake of NO compared to } \\
\text { O. No significant difference was } \\
\text { seen for isorhamnetin }(p=0.07) \\
\text { or kaempferol }(p=0.10) .\end{array}$ & $\begin{array}{l}\text { No organic certification } \\
\text { defined. The study used } \\
\text { local, known conventional } \\
\text { and organic growers (pork } \\
\text { from same litter, dairy, eggs, } \\
\text { fruit and vegetables sowed } \\
\text { and harvested within same } \\
\text { week and from similar } \\
\text { geographic location). }\end{array}$ \\
\hline $\begin{array}{c}\text { Akcay (2004) } \\
{[42]}\end{array}$ & $\begin{array}{c}\text { Turkey } \\
\text { Age: } 24-54 \text { years } \\
6 \text { male }+2 \text { female } \\
\text { Healthy }\end{array}$ & 8 & $\begin{array}{l}\text { Crossover trial. } 2 \\
\text { phases. 6-week } \\
\text { washout. }\end{array}$ & $\begin{array}{c}\text { Single dose of } \\
\text { conventional wine } \\
\text { (NO), or organic wine } \\
\text { (O). Men drank } 200 \\
\text { mL (alcohol content } 24 \\
\text { g) and women drank } \\
100 \text { mL (alcohol } \\
\text { content } 12 \text { g) over } 15 \\
\text { minutes. }\end{array}$ & $\begin{array}{l}\text { Blood samples at } 0,60 \text { and } 360 \\
\text { minutes, measured total phenol } \\
\text { content, SOD, Cat, TBARS, } \\
\text { LDL-TBARS. }\end{array}$ & $\begin{array}{l}\text { Very poorly reported results. } \\
\text { SOD increased at } 1 \text { hour }(p= \\
0.046 \text { ) and } 6 \text { hours }(p=0.028) \text { in } \\
\text { O group compared to baseline } \\
\text { (no group comparison). SOD at } \\
6 \text { hour increased in NO group } \\
\text { compared to baseline (no } \\
\text { values given). No significant } \\
\text { difference in TBARS. }\end{array}$ & $\begin{array}{l}\text { No organic certification } \\
\text { defined. "The organic wine } \\
\text { Cabernet Sauvignon (CS) } \\
\text { was obtained by defined } \\
\text { standards (certificated } \\
\text { grapes of Vitis vinifera } \\
\text { origin.." }\end{array}$ \\
\hline $\begin{array}{c}\mathrm{Lu}(2006) \\
{[43]}\end{array}$ & $\begin{array}{l}\text { United States } \\
\text { Age: } 3-11 \text { years } \\
13 \text { male }+10 \\
\text { female } \\
\text { School children }\end{array}$ & 23 & $\begin{array}{c}\text { Crossover trial. } 3 \\
\text { phases: } \\
\text { conventional diet } \\
(\mathrm{NO}) \text { days } 1-3 \text { and } \\
\text { days 9-15, organic } \\
\text { diet }(\mathrm{O}) \text { days } 4-8 .\end{array}$ & $\begin{array}{c}\text { Food items were } \\
\text { substituted for most of } \\
\text { children's } \\
\text { conventional diet, } \\
\text { including fruits, } \\
\text { vegetables and grains } \\
\text { for } 5 \text { days. Urine } \\
\text { samples (first and last } \\
\text { of the day) collected } \\
\text { for whole } 15 \text { day } \\
\text { period. }\end{array}$ & $\begin{array}{l}\text { Metabolites for selected OP } \\
\text { pesticides, pyrethroid } \\
\text { insecticides, and herbicides. }\end{array}$ & $\begin{array}{l}\text { Immediately after beginning O } \\
\text { diet, median urinary MDA and } \\
\text { TCPY decreased to } \\
\text { non-detectable levels, where } \\
\text { they remained until } \\
\text { conventional diets were } \\
\text { reintroduced }(p<0.01) \text {. No } \\
\text { effect of diets on other } \\
\text { metabolites. }\end{array}$ & $\begin{array}{l}\text { No organic certification } \\
\text { defined. "All organic food } \\
\text { items were purchased by } \\
\text { the research staff from a } \\
\text { single grocery store." }\end{array}$ \\
\hline
\end{tabular}


Table 1. Cont

\begin{tabular}{|c|c|c|c|c|c|c|c|}
\hline Ref. & Study population & $n$ & $\begin{array}{l}\text { Design and } \\
\text { Duration }\end{array}$ & Exposure/treatment & Outcome measures & Results & Definition of organic \\
\hline $\begin{array}{c}\mathrm{Lu}(2008) \\
\quad[44]\end{array}$ & $\begin{array}{l}\text { United States } \\
\text { Age: } 3-11 \text { years } \\
13 \text { male }+10 \\
\text { female } \\
\text { School children }\end{array}$ & 23 & $\begin{array}{l}\text { Crossover trial. } 3 \\
\text { phases: } \\
\text { conventional diet } \\
\text { (NO) days 1-3 and } \\
\text { days 9-15, organic } \\
\text { diet (O) days 4-8. }\end{array}$ & $\begin{array}{c}\text { Details as above, } \\
\text { including testing for } \\
15 \text { - or } \\
\text { 12-consecutive-days in } \\
\text { the summer } \\
\text { (July-August) and fall } \\
\text { (October-November), } \\
\text { respectively, and a } \\
\text { 7-consecutive-day } \\
\text { sampling period in } \\
\text { both the winter } \\
\text { (January-February) } \\
\text { and spring } \\
\text { (April-May). }\end{array}$ & $\begin{array}{l}\text { Metabolites for selected OP } \\
\text { pesticides, pyrethroid } \\
\text { insecticides, and herbicides. }\end{array}$ & $\begin{array}{l}\text { Authors observed a seasonal } \\
\text { effect on organophosphorus } \\
\text { urinary biomarker levels in this } \\
\text { cohort, and this seasonality } \\
\text { corresponds to the } \\
\text { consumption of fresh produce } \\
\text { among the children throughout } \\
\text { the year. This study is extended } \\
\text { seasonal data for the same } \\
\text { study detailed in Lu } 2006 .\end{array}$ & $\begin{array}{l}\text { No organic certification } \\
\text { defined. "All organic food } \\
\text { items were purchased by } \\
\text { the research staff from a } \\
\text { single grocery store." }\end{array}$ \\
\hline $\begin{array}{c}\text { Di Renzo (2007) } \\
\text { [45] }\end{array}$ & $\begin{array}{c}\text { Italy } \\
\text { Age: } 30-65 \text { years } \\
\text { 100\% male } \\
\text { Healthy }\end{array}$ & 10 & $\begin{array}{l}\text { Crossover trial. } 2 \\
\text { phases. } 14 \text { days } \\
\text { diet } 1 \text {, then } 14 \\
\text { days diet } 2 \text { - no } \\
\text { washout. }\end{array}$ & $\begin{array}{c}\text { Conventional } \\
\text { Mediterranean diet } \\
\text { intervention (NO), } \\
\text { followed by organic } \\
\text { Mediterranean diet } \\
\text { intervention }(\mathrm{O}) .\end{array}$ & $\begin{array}{l}\text { Plasma antioxidant (ORAC) } \\
\text { capacity. }\end{array}$ & $\begin{array}{l}\text { ORAC after NO Mediterranean } \\
\text { diet was } 2.25 \mathrm{mM} \text { TE, and } 2.75 \\
\mathrm{mM} \text { TE after O Mediterranean } \\
\text { diet. This was a significant } \\
\text { increase (21\%) after the } \\
\text { consumption of O diet. }\end{array}$ & $\begin{array}{l}\text { No organic certification } \\
\text { defined. Described as an } \\
\text { "exclusively organic" diet } \\
\text { for the organic treatment } \\
\text { arm. }\end{array}$ \\
\hline $\begin{array}{c}\text { De Lorenzo (2010) } \\
{[46]}\end{array}$ & $\begin{array}{c}\text { Italy } \\
\text { Age: } 30-65 \text { years } \\
100 \% \text { male } \\
n=100 \text { healthy; } \\
n=50 \text { stable } \\
\text { chronic kidney } \\
\text { disease (CKD) }\end{array}$ & 150 & $\begin{array}{c}\text { Crossover trial. } 2 \\
\text { phases. } \\
14 \text { days diet } 1 \text {, then } \\
14 \text { days diet } 2 \text { - no } \\
\text { washout. }\end{array}$ & $\begin{array}{c}\text { Organic } \\
\text { Mediterranean diet } \\
\text { intervention (NO), } \\
\text { followed by organic } \\
\text { Mediterranean diet } \\
\text { intervention }(\mathrm{O}) .\end{array}$ & $\begin{array}{l}\text { BMI, DXA, Hcy, serum } \\
\text { phosphorus, blood glucose } \\
\text { concentrations, lipid profile, } \\
\text { inflammatory markers, } \\
\text { microalbuminuria. }\end{array}$ & $\begin{array}{l}\text { DXA showed significant } \\
\text { differences between NO } \\
\text { Mediterranean diet and O } \\
\text { Mediterranean diet for fat mass } \\
(p<0.001) \text {, average loss of } 6.1 \\
\mathrm{~kg} \text {. Significant decrease in } \\
\text { cholesterol }(p=0.04) \text {, calcium } \\
\text { and microalbuminuria ( } p= \\
0.003) \text { after O diet only in CKD } \\
\text { patients. Inflammatory } \\
\text { parameters decreased in both } \\
\text { groups after the O diet. }\end{array}$ & $\begin{array}{c}\text { No organic certification } \\
\text { defined. Described as an } \\
\text { "exclusively organic" diet } \\
\text { for the organic treatment } \\
\text { arm. }\end{array}$ \\
\hline
\end{tabular}


Table 1. Cont

\begin{tabular}{|c|c|c|c|c|c|c|c|}
\hline Ref. & Study population & $n$ & $\begin{array}{l}\text { Design and } \\
\text { Duration }\end{array}$ & Exposure/treatment & Outcome measures & Results & Definition of organic \\
\hline $\begin{array}{c}\text { Soltoft (2011) } \\
\quad[47]\end{array}$ & $\begin{array}{l}\text { Denmark } \\
\text { Age: } 18-40 \text { years } \\
100 \% \text { male } \\
\text { Healthy }\end{array}$ & 18 & $\begin{array}{l}\text { Crossover. } \\
\text { Double-blind RCT. } \\
3 \text { phases. } 12 \text { days } \\
\text { each intervention } \\
\text { with } 2 \text { week } \\
\text { wash-out. }\end{array}$ & $\begin{array}{l}3 \times \text { treatment arms. } \\
\text { (OA: organic based on } \\
\text { livestock manure, OB: } \\
\text { organic based on } \\
\text { green manure and NO: } \\
\text { conventional with } \\
\text { mineral fertilizers) } \\
\text { grown in two } \\
\text { consecutive years } \\
\text { (year } 1 \text { and 2). Diets } \\
\text { fully controlled. }\end{array}$ & $\begin{array}{l}\text { Fasting blood samples (day } 1 \\
\text { and day } 13 \text { of each treatment } \\
\text { arm) analysed for carotenoid } \\
\text { content. }\end{array}$ & $\begin{array}{l}\text { There was no significant } \\
\text { difference in the plasma } \\
\text { carotenoid content from the } \\
\text { three different diets. There was } \\
\text { very little difference between } \\
\text { the concentrations of } \\
\text { carotenoids in the carrots across } \\
\text { growth systems, or across year } \\
\text { to year of crops. }\end{array}$ & $\begin{array}{l}\text { The organic growth systems } \\
\text { were managed in } \\
\text { compliance with the Danish } \\
\text { guidelines for organic } \\
\text { farming administered by } \\
\text { the Danish Plant } \\
\text { Directorate. }\end{array}$ \\
\hline $\begin{array}{l}\text { Toaldo (2016) } \\
{[\quad[48]}\end{array}$ & $\begin{array}{c}\text { Brazil } \\
\text { Age: } 20-55 \text { years } \\
15 \text { male }+28 \\
\text { female } \\
\text { Healthy }\end{array}$ & 24 & $\begin{array}{l}\text { Crossover. } \\
\text { Single-blind RCT. } \\
3 \text { phases. 14-day } \\
\text { washout. } 3 \text { days } \\
\text { polyphenol } \\
\text { depletion prior to } \\
\text { acute dose. }\end{array}$ & $\begin{array}{c}3 \times \text { treatment arms. } \\
\text { Single dose of } 400 \mathrm{~mL} \\
\text { of conventional juice } \\
(\mathrm{NO}) \text {, organic juice } \\
(\mathrm{O}), \text { or water. Blood } \\
\text { samples were } \\
\text { collected at } 0 \text { and } 60 \\
\text { minutes. }\end{array}$ & $\begin{array}{l}\text { GSH, Cat, SOD, Gpx, TAC, } \\
\text { glucose, and uric acid. }\end{array}$ & $\begin{array}{c}\text { GSH increased by } 8.2 \% \\
(p<0.001) \text { and } 7.0 \%(p<0.05) \\
\text { after NO and O, respectively, } \\
\text { with no significant difference } \\
\text { between juices. CAT increased } \\
22 \% \text { after } \mathrm{O}(p<0.001) . \text { SOD } \\
\text { increased } 12.9 \% \text { and } 16.3 \% \text { after } \\
\text { NO and } \mathrm{O} \text {, respectively } \\
(p<0.001) \text {. GPx increased } 6.9 \% \\
\text { and } 7.3 \% \text {, respectively, after NO } \\
\text { and O }(p<0.05) .\end{array}$ & $\begin{array}{l}\text { No organic certification } \\
\text { defined. "Two red grape } \\
\text { juices were used in this } \\
\text { study: an organic juice } \\
\text { prepared with organic } \\
\text { Bordo grapes and a } \\
\text { conventional juice prepared } \\
\text { with conventional grapes.." }\end{array}$ \\
\hline $\begin{array}{c}\text { Goen (2017) } \\
{[49]}\end{array}$ & $\begin{array}{c}\text { Switzerland } \\
\text { Age: } 46-49 \text { years } \\
1 \text { male }+1 \text { female } \\
\text { Healthy }\end{array}$ & 2 & $\begin{array}{l}\text { Crossover trial. } 2 \\
\text { phases. } 11 \text { days on } \\
\text { conventional diet, } \\
\text { followed by } 18 \\
\text { days organic diet, } \\
\text { no washout. }\end{array}$ & $\begin{array}{l}\text { Conventional diet } \\
\text { (NO) or organic diet } \\
\text { (O). Participants } \\
\text { purchased/ prepared } \\
\text { all food. Urine } \\
\text { samples taken for last } \\
4 \text { days of each } \\
\text { intervention. }\end{array}$ & $\begin{array}{l}\text { Urinary pesticide excretion, } \\
\text { including DAP, pyrethroid } \\
\text { metabolites, chlorinated } \\
\text { phenoxycarboxylic acids, } \\
\text { glyphosate, AMPA. }\end{array}$ & $\begin{array}{l}\text { This very small study }(n=2) \\
\text { shows some small but } \\
\text { statistically significant } \\
\text { reduction in some components } \\
\text { of pesticide exposure with O } \\
\text { diet. NO shows } \\
\text { organophosphate pesticides } \\
\text { and some chlorinated phenoxy } \\
\text { carboxylic acids as main } \\
\text { exposure components. }\end{array}$ & $\begin{array}{l}\text { Not defined. "participants } \\
\text { switched to exclusively } \\
\text { organic food intake" }\end{array}$ \\
\hline
\end{tabular}


Table 1. Cont.

\begin{tabular}{|c|c|c|c|c|c|c|c|}
\hline Ref. & Study population & $n$ & $\begin{array}{l}\text { Design and } \\
\text { Duration }\end{array}$ & Exposure/treatment & Outcome measures & Results & Definition of organic \\
\hline$\underset{[31]}{\operatorname{Bradman}(2015)}$ & $\begin{array}{c}\text { United States } \\
\text { Age: } 3-6 \text { years } \\
\text { 19 male }+21 \\
\text { female } \\
\text { Pre-school } \\
\text { children }\end{array}$ & 40 & $\begin{array}{l}\text { Crossover trial. } \\
\text { 3-phases: } \\
\text { conventional diet } \\
\text { (NO) days 1-4, } \\
\text { organic diet (O) } \\
\text { days 5-11, } \\
\text { conventional diet } \\
\text { (NO) days 12-16. }\end{array}$ & $\begin{array}{l}\text { Prior to study children } \\
\text { enrolled primarily } \\
\text { consumed } \\
\text { conventional diet. } \\
\text { Urine samples } \\
\text { collected over } 16 \\
\text { consecutive days. } \\
\text { Food diaries kept } \\
\text { during study phases. }\end{array}$ & $\begin{array}{l}\text { Urinary concentrations of } \\
\text { pesticides ( } 23 \text { pesticide } \\
\text { metabolites including specific } \\
\text { and nonspecific metabolites for } \\
\text { OP, pyrethrin, and pyrethroid } \\
\text { insecticides and select } \\
\text { herbicides). }\end{array}$ & $\begin{array}{l}\text { Most metabolites were below } \\
\text { LOD, mean concentrations of } 6 \\
\text { were lower during O for all } \\
\text { children, and were significant } \\
\text { for total DAPs and dimethyl } \\
\text { DAPs and } 2,4-\mathrm{D} \\
\text { (2,4-dichlorophenoxyacetic acid, } \\
\text { a herbicide), with reductions of } \\
40 \%, 49 \% \text {, and } 25 \% \text {, } \\
\text { respectively }(p<0.01) .\end{array}$ & $\begin{array}{l}\text { No organic certification } \\
\text { defined. Food for the } \\
\text { organic phase was provided } \\
\text { by the researchers according } \\
\text { to the families shopping list } \\
\text { request (to maintain diet } \\
\text { similarity). }\end{array}$ \\
\hline $\begin{array}{c}\text { Oates (2014) } \\
{[34]}\end{array}$ & $\begin{array}{c}\text { Australia } \\
\text { Age: mean } 42 \\
\text { years } \\
4 \text { male }+9 \text { female } \\
\text { Healthy }\end{array}$ & 13 & $\begin{array}{c}\text { Crossover. RCT. } 2 \\
\text { phases: } \\
\text { conventional diet } \\
\text { (NO) or } \geq 80 \% \\
\text { organic diet }(\mathrm{O}) .7 \\
\text { days per } \\
\text { intervention, no } \\
\text { washout. }\end{array}$ & $\begin{array}{c}\text { Participants } \\
\text { maintained usual } \\
\text { dietary choices and } \\
\text { sourced own food. } \\
\text { Spot morning urine } \\
\text { sample analysed on } \\
\text { day } 8 \text { of each diet. }\end{array}$ & $\begin{array}{l}\text { Urinary concentrations of } \\
\text { pesticides, including six DAP } \\
\text { metabolites of OP pesticides } \\
\text { (DMP, DMTP, DMDTP, DEP, } \\
\text { DETP and DEDTP). }\end{array}$ & $\begin{array}{l}\text { Statistically significant lower } \\
\text { levels of urinary DMP and } \\
\text { DMTP }(p<0.05) \text {, with a trend } \\
\text { for DMDTP during O phase. } \\
\text { No significant difference for } \\
\text { DEP, DETP, and DEDTP. } \\
\text { Overall pesticide results in the } \\
\text { O phase were } 89 \% \text { lower than } \\
\text { in NO phase }(p=0.013) \text {. }\end{array}$ & $\begin{array}{c}\text { Not defined. "Participants } \\
\text { were asked to consume as } \\
\text { close to } 100 \% \text { conventional } \\
\text { or organic food as possible } \\
\text { during each } 7 \text { day dietary } \\
\text { period." }\end{array}$ \\
\hline
\end{tabular}

Abbreviations: 2-AAS: 2-amino-adipic semialdehyde; AMPA: aminomethylphosphonic acid; BMI: body mass index; C: control group; CKD: chronic kidney disease; Cat: catalase; CS: cabernet sauvignon; DAP: dialkylphosphate; DEP: diethylphosphate; DETP: diethylthiophosphate; DEDTP: diethyldithiophosphate; DMDTP: dimethyldithiophosphate; DMP: dimethylphosphate; DMTP: dimethylthiophosphate; DNA: deoxyribonucleic acid; DXA: dual-energy X-ray absorptiometry; FRAP: ferric reducing ability of plasma; GPx: glutathione peroxidase; GR: glutathione reductase; GSH: glutathione; Hcy: homocysteine; LDL: low density lipoprotein; MDA: malathion; NK: natural killer; NO: non-organic group; O: organic group; OP: organophosphate; ORAC: oxygen radical absorbance capacity; RCT: randomised controlled trial; SOD: superoxide dismutase; TAC: total antioxidant capacity; TAG: triacyglycerol; TBARS: thiobarbituric acid reactive substances; TCPy: 3,5,6-trichloro-2-pyridinol; TE: trolox equivalents; TEAC: trolox equivalents antioxidant capacity; Vit: vitamin; WBC: white blood cell. 
Table 2. Data extraction table - Observational Studies.

\begin{tabular}{|c|c|c|c|c|c|c|c|}
\hline Ref & Study Population & $n$ & $\begin{array}{c}\text { Design and } \\
\text { Duration }\end{array}$ & Exposure/Treatment & Outcome Measures & Results & $\begin{array}{c}\text { Definition of } \\
\text { Organic }\end{array}$ \\
\hline Jensen (1996) [50] & $\begin{array}{c}\text { Denmark } \\
\text { Age: mean } 33 \text { years } \\
100 \% \text { male } \\
\text { Members of organic } \\
\text { farming organisations }(n \\
=55) \text { / airline company } \\
\qquad(n=141)\end{array}$ & 196 & $\begin{array}{l}\text { Cross-sectional } \\
\text { study. Analysis of } \\
\text { semen samples for } \\
\text { sperm quality in } \\
\text { male organic } \\
\text { farmers and airline } \\
\text { workers. }\end{array}$ & $\begin{array}{l}\text { Diet, working conditions, } \\
\text { health, and lifestyle were } \\
\text { assessed with questionnaire. } \\
\text { Those with }>25 \% \text { organic } \\
\text { diet defined as organic } \\
\text { group. Self-reported FFQ. }\end{array}$ & $\begin{array}{l}\text { Comparison of sperm } \\
\text { concentration, seminal } \\
\text { volume, total sperm } \\
\text { count, and sperm } \\
\text { morphology. }\end{array}$ & $\begin{array}{l}\text { Sperm concentration was } 43.1 \% \\
\text { (95\%CI } 3.2 \%-98.8 \%, p=0.033 \text { ) } \\
\text { higher among men eating } \\
\text { organically produced food. } \\
\text { Seminal volume, total sperm } \\
\text { count, and sperm morphology } \\
\text { were not different between } \\
\text { groups. This was a short report } \\
\text { and missing detail on organic diet } \\
\text { definitions between groups. }\end{array}$ & $\begin{array}{l}\text { No specific } \\
\text { definition of } \\
\text { organic. }\end{array}$ \\
\hline Juhler (1999) [51] & $\begin{array}{c}\text { Denmark } \\
\text { Age: mean } 38 \text { years } \\
100 \% \text { male } \\
\text { Organic farmers }(n=85) \\
\text { / conventional farmers } \\
\quad(n=171)\end{array}$ & 256 & $\begin{array}{l}\text { Cross-sectional } \\
\text { study. Analysis of } \\
\text { semen samples for } \\
\text { sperm quality in } \\
\text { organic vs. } \\
\text { conventional } \\
\text { farmers. }\end{array}$ & $\begin{array}{c}\text { Farmers divided into three } \\
\text { groups, according to } \\
\text { organic } \\
\text { production/proportion of } \\
\text { organic food consumption: } \\
\text { none }(\mathrm{N}, 0 \%) \text {, medium (M, } \\
1-49 \%) \text {, or a high (H, } \\
50-100 \%) \text { proportion FV } \\
\text { consumed. Self-reported } \\
\text { FFQ. }\end{array}$ & $\begin{array}{l}\text { Correlation between } \\
\text { estimated dietary } \\
\text { pesticide intakes and } \\
\text { semen parameters } \\
\text { (including sperm } \\
\text { concentration, seminal } \\
\text { volume, total sperm } \\
\text { count, and sperm } \\
\text { morphology). }\end{array}$ & $\begin{array}{l}\text { Group N showed a significantly } \\
\text { lower proportion of } \\
\text { morphologically normal } \\
\text { spermatozoa, but no difference in } \\
14 \text { other semen parameters. A } \\
\text { higher intake of five specific } \\
\text { pesticides equated with a lower } \\
\text { percentage of dead spermatozoa. } \\
\text { No other significant differences } \\
\text { were found. }\end{array}$ & $\begin{array}{l}\text { No specific } \\
\text { definition of } \\
\text { organic. }\end{array}$ \\
\hline
\end{tabular}


Table 2. Cont.

\begin{tabular}{|c|c|c|c|c|c|c|c|}
\hline Ref & Study Population & $n$ & $\begin{array}{l}\text { Design and } \\
\text { Duration }\end{array}$ & Exposure/Treatment & Outcome Measures & Results & $\begin{array}{c}\text { Definition of } \\
\text { Organic }\end{array}$ \\
\hline Chiu (2018) [52] & $\begin{array}{c}\text { United States; } \\
\text { Environment and } \\
\text { Reproductive Health } \\
\text { (EARTH) Study } \\
\text { Age: mean } 35 \text { years } \\
100 \% \text { female } \\
\text { Women attending } \\
\text { fertility clinic }\end{array}$ & 325 & $\begin{array}{l}\text { Prospective cohort. } \\
\text { Artificially assisted } \\
\text { reproduction } \\
\text { (AAR) outcomes in } \\
\text { women, including } \\
\text { pregnancy/birth } \\
\text { outcomes } \\
\text { associated with } \\
\text { high and low } \\
\text { dietary pesticide } \\
\text { exposure. }\end{array}$ & $\begin{array}{c}\text { Self-reported FFQ, prior to } \\
\text { starting AAR. A total } \\
\text { Pesticide Residue Burden } \\
\text { Score (PRBS) was calculated } \\
\text { (based on pesticide residue } \\
\text { data and organic FV intake). } \\
\text { Classifications were organic } \\
>3 \text { times/week, or } \\
\text { non-organic }<3 \text { times/week. }\end{array}$ & $\begin{array}{l}\text { Clinical outcomes } \\
\text { included implantation, } \\
\text { clinical pregnancy, live } \\
\text { birth. Early ART end } \\
\text { points included markers } \\
\text { of ovarian responses to } \\
\text { stimulation (peak } \\
\text { estradiol levels, } \\
\text { endometrial thickness, } \\
\text { oocyte development, } \\
\text { total oocytes), } \\
\text { fertilization rate, and } \\
\text { embryo quality. }\end{array}$ & $\begin{array}{l}\text { High PRBS was inversely } \\
\text { associated with probability of } \\
\text { clinical pregnancy and live birth } \\
\text { per initiated cycle. Compared } \\
\text { with women in the lowest } \\
\text { quartile of high-pesticide residue } \\
\text { FV intake (<1 serving/day), } \\
\text { women in the highest quartile } \\
(\geq 2.3 \text { servings/d) had } 18 \% \\
(95 \% C \mathrm{CI}, 5 \%-30 \%) \text { lower } \\
\text { probability of clinical pregnancy } \\
\text { and } 26 \%(95 \% \mathrm{CI}, 13 \%-37 \%) \text { lower } \\
\text { probability of live birth. No } \\
\text { association was found between } \\
\text { quartiles and early ART end } \\
\text { points. The adjusted probabilities } \\
\text { of total pregnancy loss were } 7 \% \\
\text { (95\%CI, } 3 \%-15 \%), 23 \% \text { ( } 95 \% \mathrm{CI}, \\
16 \%-33 \%), 24 \% \text { ( } 95 \% \mathrm{CI} \\
15 \%-36 \%) \text {, and } 34 \% \text { ( } 95 \% \mathrm{CI}, \\
20 \%-51 \%) \text { for women in } \\
\text { increasing quartiles of } \\
\text { high-pesticide residue FV intake. }\end{array}$ & $\begin{array}{c}\text { No specific } \\
\text { definition of } \\
\text { organic. } \\
\text { Volunteers were } \\
\text { asked to provide } \\
\text { information on } \\
\text { frequency of } \\
\text { organic FV } \\
\text { consumption }(<3 \\
\text { vs } \geq 3 \text { times/week) }\end{array}$ \\
\hline Baudry (2018) [53] & $\begin{array}{c}\text { France; Nutri-Net Santé } \\
\text { Cohort study } \\
\text { Age: mean } 44 \text { years } \\
78 \% \text { female } \\
\text { General population }\end{array}$ & 68,946 & $\begin{array}{l}\text { Prospective } \\
\text { observational } \\
\text { cohort study } \\
\text { (internet-based). } \\
\text { Followed for up to } \\
7 \text { years, looking at } \\
\text { all first primary } \\
\text { cancers diagnosed } \\
\text { between study } \\
\text { inclusion and } \\
\text { November } 2016 .\end{array}$ & $\begin{array}{l}\mathrm{FFQ} \text { and cancer data } \\
\text { (self-reported, but verified } \\
\text { with medical records in } \\
>90 \% \text { of cases). Estimated } \\
\text { intake of } 16 \text { organic } \\
\text { food/beverage items } \\
\text { recorded to determine an } \\
\text { organic score. Organic } \\
\text { quartiles: } \mathrm{Q} 4=\text { highest } \\
\text { organic food intake, Q1 } \\
\text { lowest organic food intake. }\end{array}$ & $\begin{array}{l}\text { All first primary cancers } \\
\text { diagnosed between } \\
\text { study inclusion and } \\
\text { November 2016. All } \\
\text { cancer types considered } \\
\text { cases except for basal } \\
\text { cell skin carcinoma, } \\
\text { which was not } \\
\text { considered cancer. }\end{array}$ & $\begin{array}{c}\text { High organic food scores were } \\
\text { linearly and negatively associated } \\
\text { with the overall risk of cancer } \\
\text { (HR for Q4 vs Q1, } 0.75 ; 95 \% \mathrm{CI} \text {, } \\
0.63-0.88 ; \mathrm{P} \text { for trend }=.001 \text {; } \\
\text { absolute risk reduction, } 0.6 \% \text {; HR } \\
\text { for a 5-point increase, } 0.92 ; 95 \% \\
\mathrm{CI}, 0.88-0.96 \text { ). Within individual } \\
\text { cancer types, a significantly } \\
\text { reduced HR was seen for those } \\
\text { with Q4 intake vs. Q1 for all } \\
\text { lymphomas, non-Hodgkin } \\
\text { lymphoma and post-menopausal } \\
\text { breast cancer. }\end{array}$ & $\begin{array}{l}\text { No specific } \\
\text { definition of } \\
\text { organic. } \\
\text { Consumption } \\
\text { frequency of } 264 \\
\text { food and drink } \\
\text { items used to } \\
\text { calculate organic } \\
\text { score. }\end{array}$ \\
\hline
\end{tabular}


Table 2. Cont.

\begin{tabular}{|c|c|c|c|c|c|c|c|}
\hline Ref & Study Population & $n$ & $\begin{array}{c}\text { Design and } \\
\text { Duration }\end{array}$ & Exposure/Treatment & Outcome Measures & Results & $\begin{array}{c}\text { Definition of } \\
\text { Organic }\end{array}$ \\
\hline Baudry (2018) [20] & $\begin{array}{c}\text { France; Nutri-Net Santé } \\
\text { Cohort study } \\
\text { Age: mean } 58 \text { years } \\
70 \% \text { female } \\
\text { General population }\end{array}$ & 300 & $\begin{array}{c}\text { Nested matched } \\
\text { case-control study } \\
\text { of } 300 \text { participants } \\
\text { (150 low and } 150 \\
\text { high organic food } \\
\text { consumers), with } \\
\text { available fasting } \\
\text { blood samples for } \\
\text { analysis. }\end{array}$ & $\begin{array}{l}\text { Self-reported FFQ used to } \\
\text { estimate organic food intake. } \\
\text { Low and high organic food } \\
\text { consumers were grouped } \\
\text { according to proportion of } \\
\text { organic food below } 10 \% \text { or } \\
\text { above } 50 \% \text {. The average } \\
\text { proportions of organic food } \\
\text { in the diet were } 3 \%( \pm 3) \\
\text { and } 67 \%( \pm 13) \text { in the } \\
\text { conventional and organic } \\
\text { groups, respectively. }\end{array}$ & $\begin{array}{c}\text { Plasma concentrations } \\
\text { of vitamins A and } \mathrm{a} \text { as } \\
\text { well as } 6 \text { carotenoids } \\
\text { ( } \alpha \text {-carotene, } \beta \text {-carotene, } \\
\beta \text {-cryptoxanthin, lutein, } \\
\text { zeaxanthin, and } \\
\text { lycopene), copper, } \\
\text { cadmium, magnesium, } \\
\text { iron, transferrin and } \\
\text { ferritin, fatty acid } \\
\text { composition. }\end{array}$ & $\begin{array}{l}\text { No significant differences were } \\
\text { found between the } 2 \text { groups for } \\
\alpha \text {-tocopherol and retinol, } \\
\text { cadmium, copper, ferritin or } \\
\text { transferrin. Organic consumers } \\
\text { exhibited higher plasma } \\
\text { concentrations of } \alpha \text {-carotene, } \\
\beta \text {-carotene, lutein, and zeaxanthin } \\
\text { with no differences for other } \\
\text { carotenoids. Organic consumers } \\
\text { had higher magnesium, lower } \\
\text { iron, lower palmitoleic acid, } \\
\gamma \text {-linolenic acid, and } \\
\text { docosapentaenoic acid, and } \\
\text { higher linoleic acid. }\end{array}$ & $\begin{array}{l}\text { No specific } \\
\text { definition of } \\
\text { organic. } \\
\text { Consumption } \\
\text { frequency of } 264 \\
\text { food and drink } \\
\text { items used to } \\
\text { calculate organic } \\
\text { score. }\end{array}$ \\
\hline Baudry (2019) [54] & $\begin{array}{c}\text { France; Nutri-Net Santé } \\
\text { Cohort study } \\
\text { Age: mean } 58 \text { years } \\
70 \% \text { female } \\
\text { General population }\end{array}$ & 300 & $\begin{array}{c}\text { Nested matched } \\
\text { case-control study } \\
\text { of } 300 \text { participants } \\
\text { (150 low and } 150 \\
\text { high organic food } \\
\text { consumers), with } \\
\text { available urine } \\
\text { samples for } \\
\text { analysis. }\end{array}$ & $\begin{array}{l}\text { Self-reported FFQ used to } \\
\text { estimate organic food intake. } \\
\text { Low and high organic food } \\
\text { consumers were grouped } \\
\text { according to proportion of } \\
\text { organic food below } 10 \% \text { or } \\
\text { above } 50 \% \text {. The average } \\
\text { proportions of organic food } \\
\text { in the diet were } 3 \%( \pm 3) \\
\text { and } 67 \%( \pm 13) \text { in the } \\
\text { conventional and organic } \\
\text { groups, respectively. }\end{array}$ & $\begin{array}{l}\text { Urinary pesticide and } \\
\text { metabolite } \\
\text { concentrations } \\
\text { (organophosphorus, } \\
\text { pyrethroid, and azole } \\
\text { compounds). }\end{array}$ & $\begin{array}{c}\text { Pesticide concentrations were } \\
\text { mostly below LOD. For pesticide } \\
\text { metabolites, significantly higher } \\
\text { levels of DETP, DMTP, total DAPs } \\
\text { (organophosphorus metabolites) } \\
\text { and free 3-PBA (a pyrethroid } \\
\text { metabolite) were found among } \\
\text { conventional consumers } \\
\text { compared to organic consumers, } \\
\text { with median concentration levels } \\
\text { of diethylphosphate ( } 0.196 \text { versus } \\
\text { 0.297), dimethylphosphate }(0.620 \\
\text { versus } 1.382), \text { and total } \\
\text { dialkylphosphates }(0.12 \text { versus } \\
0.16), p<0.05 \text {. }\end{array}$ & $\begin{array}{l}\text { No specific } \\
\text { definition of } \\
\text { organic. } \\
\text { Consumption } \\
\text { frequency of } 264 \\
\text { food and drink } \\
\text { items used to } \\
\text { calculate organic } \\
\text { score. }\end{array}$ \\
\hline
\end{tabular}


Table 2. Cont.

\begin{tabular}{|c|c|c|c|c|c|c|c|}
\hline Ref & Study Population & $n$ & $\begin{array}{c}\text { Design and } \\
\text { Duration }\end{array}$ & Exposure/Treatment & Outcome Measures & Results & $\begin{array}{c}\text { Definition of } \\
\text { Organic }\end{array}$ \\
\hline $\begin{array}{c}\text { Brantsæter (2016) } \\
\text { [55] }\end{array}$ & $\begin{array}{l}\text { Norway; The } \\
\text { Norwegian Mother and } \\
\text { Child Cohort Study } \\
\text { (MoBa). } 100 \% \text { female } \\
\text { Pregnant women who } \\
\text { delivered a singleton } \\
\text { male infant. }\end{array}$ & 35,107 & $\begin{array}{l}\text { Prospective cohort. } \\
\text { Pregnant women } \\
\text { at gestational } \\
\text { week } 22 \text { surveyed } \\
\text { for organic food } \\
\text { consumption with } \\
\text { results correlated } \\
\text { to prevalence of } \\
\text { male infants born } \\
\text { with hypospadias } \\
\text { or cryptorchidism. }\end{array}$ & $\begin{array}{c}\text { Self-reported FFQ collected } \\
\text { information about average } \\
\text { dietary intake since start of } \\
\text { pregnancy over six groups } \\
\text { of organically produced } \\
\text { food (vegetables, fruit, } \\
\text { bread/cereal, milk/dairy } \\
\text { products, eggs, and meat). }\end{array}$ & $\begin{array}{l}\text { Association between } \\
\text { non-organic/organic } \\
\text { food consumption } \\
\text { (never/seldom vs } \\
\text { sometimes/often/mostly) } \\
\text { and development of } \\
\text { hypospadias or } \\
\text { cryptorchidism in male } \\
\text { newborns. }\end{array}$ & $\begin{array}{l}\text { Seventy-four male newborns } \\
\text { were diagnosed with } \\
\text { hypospadias }(0.2 \%) \text {, and } 151 \text { with } \\
\text { cryptorchidism }(0.4 \%) \text {. Women } \\
\text { who consumed any organic food } \\
\text { during pregnancy were less likely } \\
\text { to give birth to a boy with } \\
\text { hypospadias (OR }=0.42 ; 95 \% \text { CI: } \\
0.25,0.70, \text { based on } 21 \text { exposed } \\
\text { cases) than women who reported } \\
\text { they never or seldom consumed } \\
\text { organic food. Associations with } \\
\text { specific organic foods were } \\
\text { strongest for vegetable (OR }= \\
0.36 ; 95 \% \text { CI: } 0.15,0.85 ; 10 \\
\text { exposed cases) and milk/dairy } \\
\text { (OR = } 0.43 ; 95 \% \text { CI: } 0.17,1.07 ; 7 \\
\text { exposed cases) consumption. No } \\
\text { association was observed for } \\
\text { consumption of organic food and } \\
\text { cryptorchidism. }\end{array}$ & $\begin{array}{l}\text { All food sold as } \\
\text { organic in Norway } \\
\text { must be certified } \\
\text { by Debio. Debio is } \\
\text { accredited organic } \\
\text { by Norwegian } \\
\text { Accreditation and } \\
\text { by IFOAM. }\end{array}$ \\
\hline $\begin{array}{c}\text { Torjusen (2016) } \\
{[56]}\end{array}$ & $\begin{array}{c}\text { Norway; The } \\
\text { Norwegian Mother and } \\
\text { Child Cohort Study } \\
\text { (MoBa) } \\
\text { Age: mean } 28 \text { years } \\
\text { 100\% female } \\
\text { Nulliparous pregnant } \\
\text { females. }\end{array}$ & 28,192 & $\begin{array}{l}\text { Prospective cohort. } \\
\text { Pregnant women } \\
\text { at gestational } \\
\text { week } 22 \text { surveyed } \\
\text { for organic food } \\
\text { consumption with } \\
\text { results correlated } \\
\text { to prevalence of } \\
\text { pre-eclampsia. }\end{array}$ & $\begin{array}{l}\text { Among the } 28,192 \text { women } \\
\text { in this study, the majority } \\
\text { reported never/rarely eating } \\
\text { organic food; } 39.8 \% \text { ate at } \\
\text { least one organic food } \\
\text { 'sometimes'; } 7 \% \text { ate at least } \\
\text { one organic food 'often'; } \\
\text { and } 1.8 \% \text { reported use of } \\
\text { any organic food 'mostly'. }\end{array}$ & $\begin{array}{l}\text { Pre-eclampsia in } \\
\text { pregnant women. }\end{array}$ & $\begin{array}{l}\text { The prevalence of pre-eclampsia } \\
\text { in the study sample was } 5.3 \%(n \\
=1,491) \text {. Women who reported } \\
\text { eating organic 'often' or 'mostly' } \\
(n=2,493,8.8 \%) \text { had lower risk of } \\
\text { pre-eclampsia than those who } \\
\text { reported 'never/rarely' or } \\
\text { 'sometimes' (crude OR }=0.76 \text {, } \\
95 \% C I 0.61,0.96 \text {; adjusted OR }= \\
0.79,95 \% \mathrm{CI} 0.62,0.99) \text {. The lower } \\
\text { risk was evident also when } \\
\text { adjusting for overall dietary } \\
\text { quality. }\end{array}$ & $\begin{array}{c}\text { No specific } \\
\text { definition of } \\
\text { organic. Frequent } \\
\text { organic } \\
\text { consumption was } \\
\text { defined as eating } \\
\text { organic food 'often' } \\
\text { for at least one of } \\
\text { the six food } \\
\text { categories. }\end{array}$ \\
\hline
\end{tabular}


Table 2. Cont.

\begin{tabular}{|c|c|c|c|c|c|c|c|}
\hline Ref & Study Population & $n$ & $\begin{array}{l}\text { Design and } \\
\text { Duration }\end{array}$ & Exposure/Treatment & Outcome Measures & Results & $\begin{array}{l}\text { Definition of } \\
\text { Organic }\end{array}$ \\
\hline $\begin{array}{c}\text { Christensen (2013) } \\
\text { [57] }\end{array}$ & $\begin{array}{c}\text { Denmark } \\
100 \% \text { female } \\
\text { Mothers of boys } \\
\text { operated on for } \\
\text { hypospadias }(n=306) \\
\text { and matching control } \\
\text { group }(n=306)\end{array}$ & 612 & $\begin{array}{l}\text { Retrospective } \\
\text { case-control study. } \\
\text { Retrospective } \\
\text { interviews of } \\
\text { organic dietary } \\
\text { habits in mothers } \\
\text { with male infant } \\
\text { born with } \\
\text { hypospadias and } \\
\text { matched controls. }\end{array}$ & $\begin{array}{l}\text { FFQ listed choice of organic } \\
\text { food items in the first } \\
\text { trimester for milk, other } \\
\text { dairy, eggs, meat, FV. } \\
\text { Responses consisted of } \\
\text { often, sometimes, rarely and } \\
\text { never. Current dietary } \\
\text { habits (up to several years } \\
\text { post-pregnancy) were taken } \\
\text { as proxy for pregnancy diet. }\end{array}$ & $\begin{array}{l}\text { Association between } \\
\text { organic food } \\
\text { consumption of } \\
\text { specified food groups } \\
\text { during pregnancy and } \\
\text { prevalence of } \\
\text { hypospadias in infant } \\
\text { sons. }\end{array}$ & $\begin{array}{l}\text { Higher OR for hypospadias was } \\
\text { found with rare or no } \\
\text { consumption of organic non-milk } \\
\text { dairy products, however, the } \\
\text { association was not statistically } \\
\text { significant after adjustment (OR } \\
=1.36,95 \% \text { CI } 0.95,1.94) \text { ). A } \\
\text { similar association was observed } \\
\text { for mothers rarely or never } \\
\text { choosing organic eggs (OR }=1.28, \\
95 \% C I 0.92,1.79 \text { ). Total organic } \\
\text { intake showed no statistically } \\
\text { significant association, however, } \\
\text { mothers who never or rarely } \\
\text { chose any organic products had } \\
\text { nonsignificant increased odds of } \\
\text { giving birth to a boy with } \\
\text { hypospadias (adjusted OR }=1.31, \\
95 \% C I 0.78,2.21) \text {. }\end{array}$ & $\begin{array}{l}\text { No specific } \\
\text { definition of } \\
\text { organic. }\end{array}$ \\
\hline Rist (2007) [58] & $\begin{array}{l}\text { Netherlands; KOALA } \\
\text { Birth Cohort } \\
\text { Age: mean } 33 \text { years } \\
100 \% \text { female } \\
\text { Breastfeeding mothers } \\
\text { with conventional (NO) } \\
\text { or alternative (O) } \\
\text { lifestyle }\end{array}$ & 312 & $\begin{array}{l}\text { Cross-sectional } \\
\text { study. Analysis of } \\
\text { breast milk for } \\
\text { fatty acid content } \\
\text { from lactating } \\
\text { women with } \\
\text { predominantly } \\
\text { organic or } \\
\text { non-organic food } \\
\text { consumption. }\end{array}$ & $\begin{array}{l}\text { FFQ at gestational week } 34 . \\
\text { Classification into four } \\
\text { groups based on the origin } \\
\text { of meat/dairy products only. } \\
\text { Organic }=>90 \% \text { organic } \\
\text { Moderate }=50-90 \% \text { organic } \\
\text { Conventional }=<50 \% \\
\text { organic }\end{array}$ & $\begin{array}{l}\text { Amount of conjugated } \\
\text { linoleic acids in breast } \\
\text { milk of lactating women, } \\
\text { measured as } \\
\text { trans-vaccenic acid } \\
\text { (TVA) and cis-9, trans-11- } \\
\text { octadecadienoic acid } \\
\text { (Rumenic). }\end{array}$ & $\begin{array}{l}\text { Rumenic acid increased in a } \\
\text { statistically significant way } \\
\text { moving from a conventional diet } \\
\text { (n=186) to a moderately organic } \\
\text { diet }(n=33) \text {, to a strict organic } \\
\text { diet }(n=37) \text {. TVA levels were } \\
\text { higher in the two mostly organic } \\
\text { quartiles than in the conventional } \\
\quad \text { or minimal groups. }\end{array}$ & $\begin{array}{l}\text { No specific } \\
\text { definition of } \\
\text { organic. Food } \\
\text { origin specified as } \\
\text { conventional or } \\
\text { organic and } \% \text { of } \\
\text { food group as }<50 \\
\%, 50-90 \% \text { or } \\
>90 \% .\end{array}$ \\
\hline
\end{tabular}


Table 2. Cont.

\begin{tabular}{|c|c|c|c|c|c|c|c|}
\hline Ref & Study Population & $n$ & $\begin{array}{l}\text { Design and } \\
\text { Duration }\end{array}$ & Exposure/Treatment & Outcome Measures & Results & $\begin{array}{l}\text { Definition of } \\
\text { Organic }\end{array}$ \\
\hline Mueller (2010) [59] & $\begin{array}{l}\text { Netherlands; KOALA } \\
\text { Birth Cohort } \\
\text { Age: mean } 33 \text { years } \\
100 \% \text { female } \\
\text { Breastfeeding mothers } \\
\text { with conventional (NO) } \\
\text { or alternative }(\mathrm{O}) \\
\text { lifestyle }\end{array}$ & 310 & $\begin{array}{l}\text { Cross-sectional } \\
\text { study. Analysis of } \\
\text { breast milk for } \\
\text { trans fatty acid } \\
\text { content from } \\
\text { lactating women } \\
\text { with } \\
\text { predominantly } \\
\text { organic or } \\
\text { non-organic food } \\
\text { consumption. }\end{array}$ & $\begin{array}{c}\text { FFQ at gestational week } 34 . \\
\text { Classification into four } \\
\text { groups based on the origin } \\
\text { of meat/dairy products only. } \\
\text { Organic }=>90 \% \text { organic } \\
\text { Moderate }=50-90 \% \text { organic } \\
\text { Conventional }=<50 \% \\
\text { organic }\end{array}$ & $\begin{array}{l}\text { Amount of trans fatty } \\
\text { acids (TFA) in breast } \\
\text { milk of lactating women, } \\
\text { measured as different } \\
\text { trans fatty acid isomers. }\end{array}$ & $\begin{array}{l}\text { Total TFA content of mothers' } \\
\text { milk in the compared groups } \\
\text { ranged between } 3 \text { and } 3.3 \% \text { of } \\
\text { total fatty acids. There were no } \\
\text { significant differences in the total } \\
\text { TFA content between groups of } \\
\text { organic vs. non-organic intake or } \\
\text { amount of dairy fat intake } \\
\text { reported. }\end{array}$ & $\begin{array}{l}\text { No specific } \\
\text { definition of } \\
\text { organic. Food } \\
\text { origin specified as } \\
\text { conventional or } \\
\text { organic and } \% \text { of } \\
\text { food group as }<50 \\
\%, 50-90 \% \text { or } \\
>90 \% .\end{array}$ \\
\hline $\begin{array}{c}\text { Kummeling (2008) } \\
\text { [60] }\end{array}$ & $\begin{array}{l}\text { Netherlands; KOALA } \\
\text { birth Cohort } \\
\text { Age: } 2 \text { years } \\
\text { Infants with } \\
\text { non-organic (NO) ( } n= \\
\text { 2,135), or organic diet } \\
\text { (O) }(n=463)\end{array}$ & 2598 & $\begin{array}{l}\text { Prospective cohort. } \\
\text { Mothers of infants } \\
\text { surveyed about } \\
\text { child's organic } \\
\text { food consumption } \\
\text { and allergy } \\
\text { symptoms at } 3,7, \\
12 \text { and } 24 \text { months } \\
\text { of age. }\end{array}$ & $\begin{array}{c}\text { Parents completed FFQ at } \\
\text { each time-point. Infants diet } \\
\text { classified as: 'conventional' } \\
\text { ( }<50 \% \text { organic); 'moderately } \\
\text { organic' (50-90\% organic); } \\
\text { 'strictly organic' (>90\% } \\
\text { organic). }\end{array}$ & $\begin{array}{l}\text { Association between } \\
\text { allergic symptoms } \\
\text { reported by parents } \\
\text { (including eczema, } \\
\text { wheeze occurrence, } \\
\text { rash) and intake of } \\
\text { organic vs. conventional } \\
\text { foods; IgE antibodies } \\
\text { measured in a subset of } \\
\text { children }(n=815) \text {. }\end{array}$ & $\begin{array}{c}\text { Consumption of organic dairy } \\
\text { products was associated with } \\
\text { lower eczema risk (OR }=0 \cdot 64, \\
95 \% \text { CI } 0 \cdot 44,0 \cdot 93) \text {, but there was } \\
\text { no association for development of } \\
\text { eczema, wheeze or atopic } \\
\text { sensitisation. No statistically } \\
\text { significant associations were } \\
\text { observed between organic food } \\
\text { consumption and recurrent } \\
\text { wheeze (OR }=0.51,95 \% \text { CI } 0 \cdot 26 \text {, } \\
\text { 0.99) during the first } 2 \text { years of } \\
\text { life. }\end{array}$ & $\begin{array}{l}\text { In the Netherlands } \\
\text { 'organic' products } \\
\text { include } \\
\text { biodynamic } \\
\text { production, which } \\
\text { carry the } \\
\text { registered 'EKO' } \\
\text { certification. }\end{array}$ \\
\hline Stenius (2011) [61] & $\begin{array}{c}\text { Sweden; ALLADIN } \\
\text { Study } \\
\text { Age: foetal period (2 } 2^{\text {nd }} \\
\text { trimester of mother })-24 \\
\text { months }\end{array}$ & 330 & $\begin{array}{l}\text { Prospective cohort. } \\
\text { Anthroposophic or } \\
\text { non-anthroposophic } \\
\text { families followed } \\
\text { for development } \\
\text { of allergic } \\
\text { sensitisation in } \\
\text { children, } \\
\text { correlated with } \\
\text { lifestyle factors } \\
\text { (including organic } \\
\text { food choice). }\end{array}$ & $\begin{array}{l}\text { FFQ completed by pregnant } \\
\text { women in } 2^{\text {nd }} \text { trimester. } \\
\text { Child followed for allergic } \\
\text { sensitisation to } 24 \text { months. } \\
\text { Organic food consumption } \\
\text { in AL group was } 80 \% \text { and } \\
5 \% \text { in CL group. }\end{array}$ & $\begin{array}{l}\text { IgE in cord blood and } \\
\text { sensitisation to common } \\
\text { allergens and total IgE } \\
\text { at } 6,12 \text {, and } 24 \text { months } \\
\text { of age. }\end{array}$ & $\begin{array}{l}\text { Children of families with AL had } \\
\text { a markedly decreased risk of } \\
\text { sensitisation during the first } 2 \\
\text { years of life compared with } \\
\text { children of CL families with } \\
\text { adjusted OR }=0.25 \text { (95\%CI } 0.10, \\
0.64), p=0.004 \text {. Children from } \\
\text { families with a partly } \\
\text { anthroposophic lifestyle had } \\
\text { similar result with adjusted OR }= \\
0.31 \text { ( } 95 \% \text { CI } 0.15,0.54), p=0.002 \text {. }\end{array}$ & $\begin{array}{l}\text { No specific } \\
\text { definition of } \\
\text { organic. } \\
\text { Organic/biodynamic } \\
\text { diet evaluated as } \\
\text { one of many } \\
\text { lifestyle questions, } \\
\text { with no detail of } \\
\text { how this was } \\
\text { quantified. }\end{array}$ \\
\hline
\end{tabular}


Table 2. Cont.

\begin{tabular}{|c|c|c|c|c|c|c|c|}
\hline Ref & Study Population & $n$ & $\begin{array}{l}\text { Design and } \\
\text { Duration }\end{array}$ & Exposure/Treatment & Outcome Measures & Results & $\begin{array}{l}\text { Definition of } \\
\text { Organic }\end{array}$ \\
\hline Buscail (2015) [62] & $\begin{array}{l}\text { France; PELAIGE } \\
\text { mother-child cohort } \\
\text { Age: foetal period (from } \\
\text { gestation) - } 24 \text { months }\end{array}$ & 1505 & $\begin{array}{l}\text { Prospective } \\
\text { observational } \\
\text { cohort study. } \\
\text { Mothers } \\
\text { consumption of } \\
\text { organic food } \\
\text { mid-pregnancy } \\
\text { and when infant is } \\
2 \text { years, correlated } \\
\text { to episodes of } \\
\text { otitis media. }\end{array}$ & $\begin{array}{l}\text { Pregnant women completed } \\
\text { questionnaires reporting } \\
\text { domestic use of pesticides } \\
\text { and consumption of organic } \\
\text { diet during pregnancy at } 19 \\
\text { weeks of gestation and } \\
\text { again at age } 2 \text { of infant. } \\
\text { Children were assessed for } \\
\text { otitis media during early } \\
\text { childhood. }\end{array}$ & $\begin{array}{l}\text { Episodes of otitis media } \\
\text { (OM) and recurrent OM } \\
\text { in children. Urinary } \\
\text { samples to measure } \\
\text { pesticides }(n=248) \text {. } \\
\text { Associations between } \\
\text { pesticide measurements } \\
\text { and OM. }\end{array}$ & $\begin{array}{c}\text { Children whose mothers reported } \\
\text { an organic diet during pregnancy } \\
\text { had a reduced risk of OM (at least } \\
\text { one episode, } p \text { trend }=0.01) \text {. No } \\
\text { association was found between } \\
\text { any outcome and residential } \\
\text { proximity to crops. The presence } \\
\text { in maternal urine of dealkylated } \\
\text { triazine metabolites (herbicide) } \\
\text { was positively associated with } \\
\text { recurrent OM }(\mathrm{OR}=2.12 \text { (1.01 to } \\
4.47)) .\end{array}$ & $\begin{array}{l}\text { No specific } \\
\text { definition of } \\
\text { organic. Fruit, } \\
\text { vegetables and } \\
\text { cereals from a } \\
\text { non-organic diet } \\
\text { were selected as } \\
\text { proxies for } \\
\text { insecticide } \\
\text { exposure. }\end{array}$ \\
\hline $\begin{array}{l}\text { Kesse-Guyot } \\
\text { (2017) [63] }\end{array}$ & $\begin{array}{c}\text { France; Nutri-Net Santé } \\
\text { Cohort study } \\
\text { Age: mean } 45 \text { years } \\
78 \% \text { Female } \\
\text { General population }\end{array}$ & 62,224 & $\begin{array}{l}\text { Prospective cohort } \\
\text { (internet-based). } \\
\text { Followed for up to } \\
10 \text { years, looking } \\
\text { at body weight } \\
\text { change, risk of } \\
\text { overweight or } \\
\text { obesity and } \\
\text { consumption of } \\
\text { organic food. }\end{array}$ & $\begin{array}{l}\text { Self-reported FFQ and } \\
\text { anthropometric data } \\
\text { completed annually } \\
\text { (average } 3.1 \text { year follow-up). } \\
\text { Estimated intake of } 16 \\
\text { organic food/beverage items } \\
\text { recorded to determine an } \\
\text { organic score (OS). Organic } \\
\text { diet quartiles: } \mathrm{Q} 4=\text { highest } \\
\text { rate of organic food } \\
\text { consumption, } \mathrm{Q} 1=\text { lowest } \\
\text { rate of organic food } \\
\text { consumption. }\end{array}$ & $\begin{array}{c}\text { Correlation between the } \\
\text { OS and change in BMI } \\
\text { during follow-up and } \\
\text { risk of overweight and } \\
\text { obesity. }\end{array}$ & $\begin{array}{l}\text { Lower BMI increase was } \\
\text { observed across quartiles of the } \\
\text { OS (mean difference Q4 v.Q1 = } \\
-0.16(95 \% \mathrm{CI}-0.32,-0.01) \text {. An } \\
\text { increase in the OS was associated } \\
\text { with a lower risk of overweight } \\
\text { and obesity (among } \\
\text { non-overweight and non-obese } \\
\text { participants at inclusion): OR for } \\
\mathrm{Q} 4 \mathrm{v} \text {. Q1 were } 0.77(95 \% \mathrm{CI} 0.68, \\
0.86 \text { ) and } 0.69(95 \% \mathrm{CI} 0.58,0.82) \text {, } \\
\text { respectively. The association } \\
\text { remained strong and highly } \\
\text { significant, with a reduction in } \\
\text { the risk of obesity of } 37 \% \text { at } \\
\text { follow-up. A similar association } \\
\text { was observed for overweight, } \\
\text { although the strength of the } \\
\text { association was smaller. }\end{array}$ & $\begin{array}{l}\text { No specific } \\
\text { definition of } \\
\text { organic. } \\
\text { Consumption } \\
\text { frequency of } 264 \\
\text { food and drink } \\
\text { items used to } \\
\text { calculate organic } \\
\text { score. }\end{array}$ \\
\hline
\end{tabular}


Table 2. Cont.

\begin{tabular}{|c|c|c|c|c|c|c|c|}
\hline Ref & Study Population & $n$ & $\begin{array}{c}\text { Design and } \\
\text { Duration }\end{array}$ & Exposure/Treatment & Outcome Measures & Results & $\begin{array}{c}\text { Definition of } \\
\text { Organic }\end{array}$ \\
\hline Baudry (2017) [64] & $\begin{array}{c}\text { France; Nutri-Net Santé } \\
\text { Cohort study } \\
\text { Age: mean } 45 \text { years } \\
78 \% \text { Female } \\
\text { General population }\end{array}$ & 8174 & $\begin{array}{l}\text { Cross-sectional } \\
\text { analysis of } \\
\text { proportion of } \\
\text { organic food in the } \\
\text { diet (overall and } \\
\text { by food group) } \\
\text { and prevalence of } \\
\text { metabolic } \\
\text { syndrome. }\end{array}$ & $\begin{array}{c}\text { Participants filled out a } \\
\text { self-administered FFQ, } \\
\text { including } 264 \text { food and } \\
\text { beverage items. Separated } \\
\text { into tertiles of organic food } \\
\text { consumption. }\end{array}$ & $\begin{array}{l}\text { Correlation between } \\
\text { level of organic food } \\
\text { intake and prevalence of } \\
\text { metabolic syndrome. }\end{array}$ & $\begin{array}{l}\text { Higher organic food } \\
\text { consumption was associated with } \\
\text { a lower probability of metabolic } \\
\text { syndrome, being negatively } \\
\text { associated with prevalence, } 0.69 \\
(95 \% \text { CI } 0.61,0.78) \text { when } \\
\text { comparing the third tertile of } \\
\text { proportion of organic food in the } \\
\text { diet with the first one }(p<0.0001) \text {. }\end{array}$ & $\begin{array}{l}\text { No specific } \\
\text { definition of } \\
\text { organic. } \\
\text { Consumption } \\
\text { frequency of } 264 \\
\text { food and drink } \\
\text { items used to } \\
\text { calculate organic } \\
\text { score. }\end{array}$ \\
\hline $\begin{array}{c}\text { Bradbury (2014) } \\
\text { [65] }\end{array}$ & $\begin{array}{c}\text { United Kingdom; The } \\
\text { Million Women Study } \\
\text { Age: mean } 59 \text { years } \\
100 \% \text { Female } \\
\text { General population }\end{array}$ & 623,080 & $\begin{array}{l}\text { Prospective cohort. } \\
\text { Study correlates } \\
\text { frequency of } \\
\text { organic food } \\
\text { intake to cancer } \\
\text { incidence in } \\
\text { women, followed } \\
\text { on average for } 9.3 \\
\text { years. }\end{array}$ & $\begin{array}{c}\text { Women without cancer at } \\
\text { baseline completed a } \\
\text { questionnaire asking 'Do } \\
\text { you eat organic food?' with } \\
\text { four possible responses: } \\
\text { 'never, sometimes, usually, } \\
\text { and always.' Repeated at } \\
\text { follow-up (on average 9.3 } \\
\text { yr). }\end{array}$ & $\begin{array}{l}\text { Association of organic } \\
\text { diet with cancer, } \\
\text { including all cancers } \\
\text { combined (except } \\
\text { non-melanoma skin } \\
\text { cancer), oral, } \\
\text { oesophageal, stomach, } \\
\text { colorectum, pancreas, } \\
\text { lung, malignant } \\
\text { melanoma, breast, } \\
\text { endometrium, ovary, } \\
\text { kidney, bladder, brain, } \\
\text { non-Hodgkin } \\
\text { lymphoma, multiple } \\
\text { myeloma, and } \\
\text { leukaemia. }\end{array}$ & $\begin{array}{c}\text { At baseline, } 30 \%, 63 \% \text { and } 7 \% \text { of } \\
\text { women reported never, } \\
\text { sometimes, or usually/always } \\
\text { eating organic food, respectively. } \\
\text { Consumption of organic food } \\
\text { was not associated with a } \\
\text { reduction in the incidence of all } \\
\text { cancer ( } n=53,769 \text { cases in total) } \\
\text { (RR for usually/always vs never }= \\
1.03,95 \% C I 0.99,1.07) \text {, soft tissue } \\
\text { sarcoma (RR }=1.37,95 \% C I 0.82, \\
2.27) \text {, or breast cancer (RR }=1.09 \text {, } \\
95 \% \text { CI } 1.02,1.15), \text { but was } \\
\text { associated with reduced risk for } \\
\text { non-Hodgkin lymphoma (RR = } \\
0.79,95 \% C I 0.65,0.96) .\end{array}$ & $\begin{array}{l}\text { No specific } \\
\text { definition of } \\
\text { organic. }\end{array}$ \\
\hline $\begin{array}{c}\text { McGuire (2016) } \\
\text { [66] }\end{array}$ & $\begin{array}{c}\text { United States } \\
\text { Age: mean } 29 \text { years } \\
100 \% \text { female } \\
\text { Breastfeeding women } \\
1-3 \text { months postpartum }\end{array}$ & 41 & $\begin{array}{l}\text { Cross-sectional } \\
\text { study. Single milk } \\
\text { and urine sample } \\
\text { from each woman } \\
\text { to assess level of } \\
\text { pesticides. }\end{array}$ & $\begin{array}{l}5 \text { question survey that } \\
\text { documented potential } \\
\text { glyphosate exposure from } \\
\text { environment and diet. } 42 \% \\
\text { of the women identified as } \\
\text { having "strictly or mainly } \\
\text { organic food choices" }\end{array}$ & $\begin{array}{l}\text { Glyphosate and AMPA } \\
\text { concentrations in } \\
\text { human milk, correlated } \\
\text { with pesticide excretion } \\
\text { in urine samples. }\end{array}$ & $\begin{array}{l}\text { Glyphosate and AMPA were not } \\
\text { detectable in milk samples. There } \\
\text { were no significant effects of } \\
\text { consuming organic over } \\
\text { conventional foods or living } \\
\text { on/near a farm compared with } \\
\text { living in an urban/suburban } \\
\text { region on concentrations of } \\
\text { glyphosate }(p=0.1870 \text { and } 0.8773, \\
\text { respectively), or AMPA in urine } \\
(p=0.1414 \text { and } 0.2525 \\
\text { respectively). }\end{array}$ & $\begin{array}{l}\text { No specific } \\
\text { definition of } \\
\text { organic. Food } \\
\text { intake was } \\
\text { self-reported as } \\
\text { either mainly } \\
\text { organic or mainly } \\
\text { conventional. }\end{array}$ \\
\hline
\end{tabular}


Table 2. Cont.

\begin{tabular}{|c|c|c|c|c|c|c|c|}
\hline Ref & Study Population & $n$ & $\begin{array}{l}\text { Design and } \\
\text { Duration }\end{array}$ & Exposure/Treatment & Outcome Measures & Results & $\begin{array}{c}\text { Definition of } \\
\text { Organic }\end{array}$ \\
\hline Curl (2003) [33] & $\begin{array}{c}\text { United States } \\
\text { Age: } 2-5 \text { years } \\
56 \% \text { Male } \\
\text { Children }\end{array}$ & 39 & $\begin{array}{l}\text { Cross-sectional } \\
\text { study. Level of } \\
\text { organic food (\%) in } \\
\text { diet correlated to } \\
\text { pesticide excretion } \\
\text { in urine. Food } \\
\text { frequency data } \\
\text { and urine samples } \\
\text { were collected. }\end{array}$ & $\begin{array}{c}\text { Parents of children } \\
\text { interviewed about diet, } \\
\text { health information and } \\
\text { pesticide use, with } 2 \text { day } \\
\text { food diary completed on } \\
\text { day of child's urine } \\
\text { sampling. Diet reported as } \\
\text { mostly organic or mostly } \\
\text { conventional. }\end{array}$ & $\begin{array}{l}\text { 24-hour urine samples } \\
\text { measured for urinary } \\
\text { DAP or DMP } \\
\text { concentrations. }\end{array}$ & $\begin{array}{l}\text { The median total DMP } \\
\text { concentration was approximately } \\
\text { six times higher for children with } \\
\text { conventional diets than for } \\
\text { children with organic diets }(0.17 \\
\text { and } 0.03 \mu \text { mol/L; } p=0.0003) ; \\
\text { mean concentrations differed by a } \\
\text { factor of nine }(0.34 \text { and } 0.04 \\
\mu \mathrm{mol} / \mathrm{L}) \text {. }\end{array}$ & $\begin{array}{l}\text { No specific } \\
\text { definition of } \\
\text { organic. }>75 \% \text { of } \\
\text { dietary intake as } \\
\text { organic or } \\
\text { conventionally } \\
\text { separated the two } \\
\text { groups. }\end{array}$ \\
\hline Curl (2015) [32] & $\begin{array}{c}\text { United States } \\
\text { Age: } 45-84 \text { years } \\
50 \% \text { Female } \\
\text { Adults with subclinical } \\
\text { cardiovascular disease }\end{array}$ & 6814 & $\begin{array}{l}\text { Cross-sectional } \\
\text { study. Food } \\
\text { frequency data } \\
\text { and urine samples } \\
\text { were collected, } \\
\text { correlating organic } \\
\text { intake to urinary } \\
\text { excretion of } \\
\text { pesticides. }\end{array}$ & $\begin{array}{l}\text { Diet was reported as mostly } \\
\text { organic or conventional. } \\
\text { Participants were asked } \\
\text { about their "usual" intake } \\
\text { of specific foods and } \\
\text { beverages "over the past } \\
\text { year." Average pesticide } \\
\text { intake was then calculated. }\end{array}$ & $\begin{array}{l}\text { Correlation between } \\
\text { pesticide intake and } \\
\text { excretion of pesticides in } \\
\text { urine. Sub-group } \\
\text { analysis of urine } \\
\text { samples for pesticides ( } n \\
=240) .\end{array}$ & $\begin{array}{c}\text { Among conventional consumers, } \\
\text { increasing tertile of estimated } \\
\text { dietary organophosphate } \\
\text { exposure was associated with } \\
\text { higher DAP concentrations }(p< \\
\text { 0.05). DAP concentrations were } \\
\text { also significantly lower in groups } \\
\text { reporting more frequent } \\
\text { consumption of organic produce } \\
(p<0.02) .\end{array}$ & $\begin{array}{l}\text { No specific } \\
\text { definition of } \\
\text { organic. Foods } \\
\text { eaten are } \\
\text { correlated to US } \\
\text { Dept Agriculture } \\
\text { data on pesticide } \\
\text { residues and a } \\
\text { pesticide exposure } \\
\text { number assigned. }\end{array}$ \\
\hline
\end{tabular}

Abbreviations: AAR: artficially assisted reproduction; AL: anthroposophic lifestyle; ART: assisted reproductive technology; AMPA: aminomethylphosphonic acid; BMI: body mass index; CL: conventional lifestyle; DAP: dialkylphosphate; DETP: diethylthiophosphate; DMP: dimethylphosphate; DMTP: dimethylthiophosphate; FFQ: food frequency questionnaire; FV: fruits and vegetables; HR: hazard ratio; LOD: limit of detection; NO: non-organic group; O: organic group; OM: otitis media; OS: organic score; PBA: 3-phenoxybenzoic acid; PRBS: pesticide residue burden score; TFA: trans-fatty acid; TVA: trans-vaccenic acid; Vit: vitamin. 


\subsection{Assessment of Risk of Bias}

The Cochrane Risk of Bias Assessment Tool was used to assess likelihood of bias in each clinical trial publication [67]. The Newcastle-Ottawa Quality Assessment Form for Cohort Studies was used to assess the likelihood of bias in cohort studies, and the Specialist Unit for Review Evidence (SURE) checklist was used for the critical appraisal of cross-sectional studies [68,69]. All assessments were conducted by at least two authors, with differences settled by discussion. Summary tables detailing results of bias assessments are presented in Supplementary Figure S2.

\section{Results}

\subsection{Study Selection and Characteristics}

The Preferred Reporting Items for Systematic Reviews and Meta-Analyses (PRISMA) flow diagram detailing the article selection process is shown in Figure 1. Searches identified 4329 potentially relevant articles, of which 4234 were excluded after initial screening of title and/or abstract. The remaining 95 full-text publications were assessed, of which a further 60 publications were excluded.

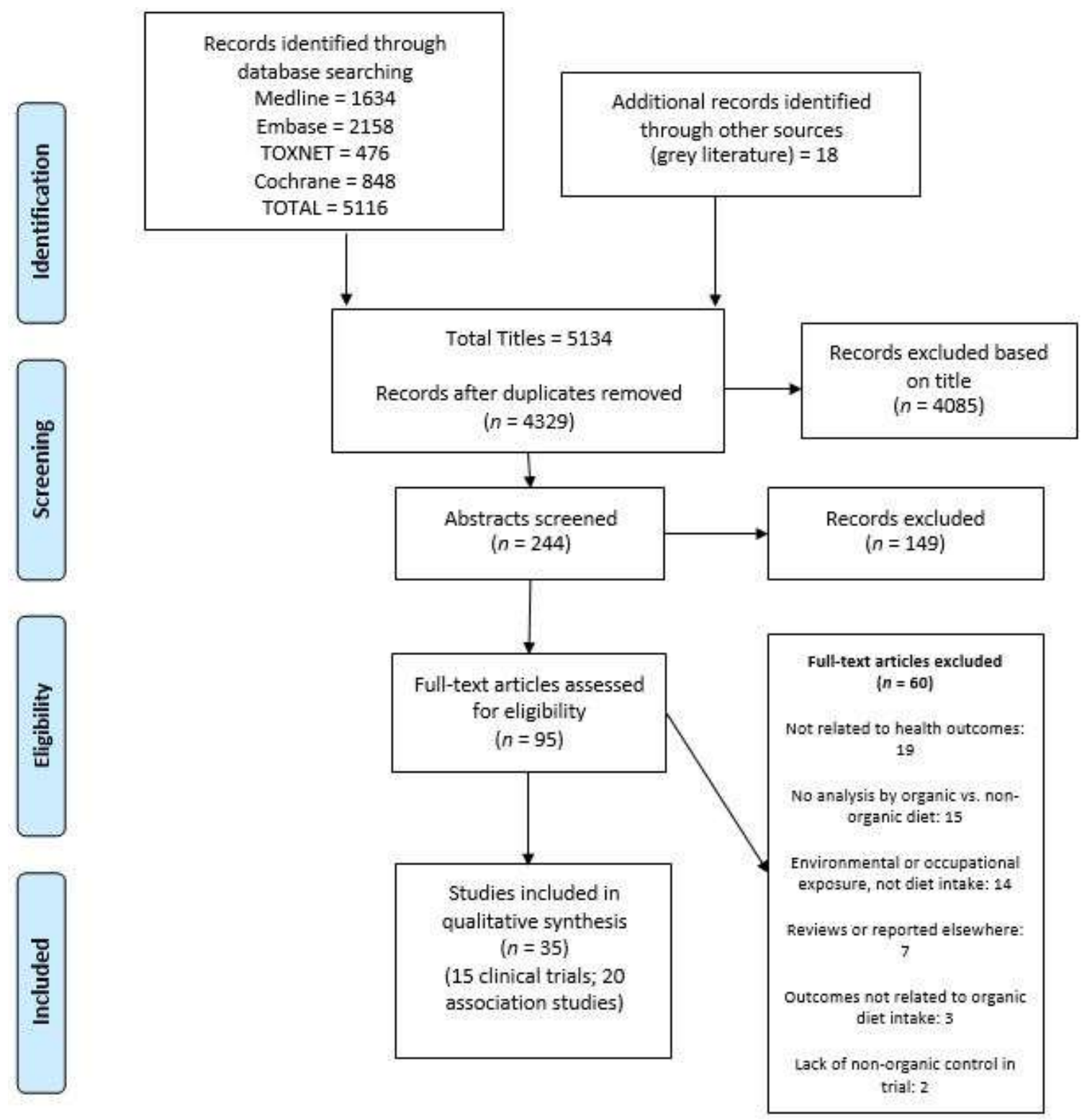

Figure 1. PRISMA flow diagram of study selection [36]. 
Thirty-five papers met the criteria for inclusion in this review. Of these, 15 publications reported on 13 clinical trials - three of which were parallel-arm randomised controlled trials (RCT), with the remaining studies utilising a crossover design. In observational studies, 20 publications reported on 13 cohorts. The studies were all published in English. The majority of the clinical trials were conducted in Europe-Germany (2), Denmark (2), Italy (2), France (1), and Switzerland (1), with other countries including: the United States (2), Turkey (1), Brazil (1), and Australia (1). Observational research studies were on cohorts from the United States, United Kingdom, Norway, France, Denmark, Netherlands, and Sweden.

\subsection{Clinical Trials (Single Food/Drink Item Substitution)}

Several studies investigated the effect of replacing a single non-organic food or drink item with its organic counterpart. Three of the trials utilised an acute dose setting (red wine, apples or grape juice) in a crossover design $[40,42,48]$, while others were based on the daily consumption of the food item (tomatoes and derived purees, carrots or apples) for a period of 2-4 weeks [37-39]. Those studies looking at nutrient levels (i.e., carotenoids, polyphenols) [37-39] in biological samples (blood or urine), did not find any significant differences in the levels of these markers as a result of the organic intervention.

Other single-item substitution studies measured antioxidant capacity, or DNA damage in biological samples [38-40,42,48]. There were no significant between-group differences in these biomarkers in any of the studies.

\subsection{Clinical Trials (Whole Diet Substitution)}

Eight crossover trials (reported in nine publications) investigated the effect of whole diet replacement from conventional to organic (or at least $>80 \%$ in one study) for a time period ranging from 4 or 5 days in children [31,43,44] to up to 22 days in adult populations $[34,41,45-47,49]$.

Four of these trials (two in children and two in adults) measured changes in pesticide excretion through urine $[31,34,43,44,49]$. All of these trials demonstrated a significant difference in the amount of pesticide metabolites excreted during the different phases of the diet interventions. The reduction was, in most cases, dramatic (up to $90 \%$ reduction during organic phase) and occurred within a short time frame of only a few days.

The remaining trials were all conducted in adult populations and measured antioxidant capacity and flavonoid excretion [41]; carotenoids [47]; or antioxidant capacity, changes to body composition, lipids and inflammatory markers [45,46].

Similar to the results from clinical trials replacing single food items, individual flavonoid and carotenoid excretion appeared to reflect the content of the foods consumed (i.e., a higher quercetin, carotenoid and kaempferol level was shown in organic produce in comparison to conventional produce given as part of the diets, and this was reflected in the urinary output) [41,47].

Two studies completed by the same research group in Italy looked at the effects of a Mediterranean diet intervention (non-organic phase followed by organic phase). An initial pilot study of 10 people [45] and a following larger cohort study of 150 people (100 healthy and 50 with chronic kidney disease (CKD)) [46] provided a two-stage intervention, with a controlled Mediterranean diet (MD) for 14 days followed by the same diet for a further 14 days using organic rather than conventional foodstuffs.

The pilot study found an increased antioxidant effect (from 2.25 to $2.75 \mathrm{mM}$ trolox equivalents) after 14 days MD and after 14 days organic MD, respectively, with no baseline measure provided. The authors also showed a generally higher antioxidant level in the organic foods eaten in comparison to non-organic. In the larger study, in both healthy and CKD patients there was a highly significant effect on body weight reduction and improved body composition seen through dual-energy X-ray absorptiometry (DXA) and bio-impedance analysis (BIA) between the two time points (end of conventional MD and end of organic MD). Inflammatory markers (hs-CRP, IL-1, IL-6, IFN- $\gamma$ and homocysteine) all showed 
a statistically significant decrease between the same time-points for the healthy group, whilst only hs-CRP and homocysteine were significantly decreased in the CKD group.

\subsection{Observational cohort studies}

From a total of 20 publications including 13 cohorts, seven prospective cohorts were identified, with the majority involving mother/child pairs. These included the Norwegian Mother and Child Cohort Study [55,56]; KOALA Birth Cohort [58-60]; ALLADIN study [61]; PELAIGE Mother-Child Cohort [62] and the EARTH study [52]. Two adult-only cohorts involved development of cancer incidence in the Million Women Study [65], and self-reported health factors in the Nutri-Net Santé Cohort Study $[20,53,63,64]$. A retrospective case-control study in a mother-child cohort was also included [57].

Several of the identified studies provided cross-section data only. These include comparisons of organic and conventional diets on sperm quality/content [50,51]; breast milk composition [66]; and urinary pesticide excretion $[32,33]$.

For ease of reporting, all of the observational studies have been separated into subject areas. Firstly, looking at potential influence on foetal development (effect on sperm, fertility, and birth defects, pre-eclampsia); breast milk studies; development of allergies in children; urinary pesticide excretion; cancer development incidence; and changes in nutritional biomarkers in adults.

\subsubsection{Sperm and Fertility}

Two investigations examined the association between sperm health in Danish organic farmers. The first compares the organic farmers to non-organic farmers and shows a significantly lower proportion of morphologically normal spermatozoa in the non-organic group, but no significant difference in relation to 14 other semen parameters [51]. The other compares the organic farmers to a control group of airline pilots, finding a higher sperm concentration among organic farmers (increased by $43.1 \%, 95 \%$ CI 3.2 to 98.8\%), with no differences seen in seminal volume, total sperm count, and sperm morphology [50].

The Environment and Reproductive Health (EARTH) study examined associations between high or low dietary pesticide exposure in a group of women using assisted reproduction technology (ART) at the Massachusetts General Hospital Fertility Center [52]. They compared pregnancy/birth outcomes from 325 women (contributing 541 ART cycles) against a dietary pesticide score. They found high-pesticide residue fruit and vegetable (FV) intake was inversely associated with probability of clinical pregnancy and live birth per initiated cycle. Compared with women in the lowest quartile of high-pesticide residue FV intake ( $<1$ serving/day), women in the highest quartile ( $\geq 2.3$ servings/day) had $18 \%$ (95\%CI 5\%-30\%) lower probability of clinical pregnancy and 26\% (95\%CI 13\%-37\%) lower probability of live birth. High-pesticide residue FV intake was positively associated with probability of total pregnancy loss.

\subsubsection{Mother-Child cohorts}

The Norwegian Mother and Child Cohort Study (MoBa) investigated associations between an organic diet and conventional diet during pregnancy and the development of pregnancy complications, including pre-eclampsia [56] and incidence of the rare reproductive abnormalities in infant boys-hypospadias or cryptorchidism [55]. Women who reported to have eaten organic vegetables 'often' or 'mostly' ( $n=2493,8.8 \%$ of study-sample) were found to have a lower risk of pre-eclampsia than those who reported 'never/rarely' or 'sometimes' (OR $=0.76,95 \% \mathrm{CI} 0.61,0.96)$. A lower prevalence of hypospadias with any organic consumption, in particular organic vegetables, was found, with no difference for cryptorchidism. This prospective study included 35,107 mothers of male infants in Norway, with organic food in six food groups assessed by food frequency questionnaires (FFQ) [55]. Whole diet composition was considered using slightly different methods in each of these analyses; therefore, residual confounding may exist between the results reported. In a smaller case-control study, retrospective data were collected from mothers of 306 infant males who were 
operated on for hypospadias matched to 306 mothers of healthy infant males in Denmark. No difference was found for total organic consumption, but increased odds for hypospadias were found specifically when non-organic milk/dairy consumption was combined with frequent consumption of high-fat dairy products (adjusted OR $=2.18,95 \%$ CI 1.09, 4.36) [57].

The PELAIGE study in France $(n=1505)$ was a prospective cohort study that examined the incidence of otitis media during early childhood, finding frequent intake of organic diet during pregnancy was associated with decreased risk of having at least one episode of otitis media $(\mathrm{OR}=0.69$, $95 \%$ CI $0.47,1.00)$ [62]. A sub-group analysis measuring pesticide residues in urine, found the presence of dealkylated triazine metabolites was positively associated with recurrent otitis media $(\mathrm{OR}=2.12$, 95\%CI 1.01, 4.47).

The influence of organic food consumption as part of an anthroposophical lifestyle in pregnancy and early childhood has been discussed following two major studies - the KOALA birth cohort in the Netherlands [60,70,71], and the ALADDIN birth cohort in Sweden [61]. In the KOALA cohort $(n=2764)$, consumption of organic dairy products was associated with lower eczema risk $(\mathrm{OR}=0.64$, $95 \%$ CI $0.44,0.93)$, but there was no association for other food types or overall organic content of diet with the development of eczema, wheeze or atopic sensitisation. No statistically significant associations were observed between organic food consumption and recurrent wheeze (OR $=0.51,95 \%$ CI $0.26,0.99$ ) during the first 2 years of life [60]. In the ALADDIN study $(n=330)$, a markedly decreased risk of sensitisation during the first 2 years of life was seen in children of anthroposophic families compared with children of non-anthroposophic families with adjusted OR of 0.25 (95\%CI 0.10, 0.64, $p=0.004$ ) [61].

It is important to note that organic food consumption is only one of several food-specific differences that are a key part of the anthroposophic lifestyle (see discussion).

\subsubsection{Early Childhood}

Minimal changes were seen in breastmilk composition in the KOALA birth cohort study, with increased rumenic acid and a trend for increased trans-vaccenic acid in quartiles of highest organic consumption [58]. No difference was seen in trans fatty acid content within the same cohort [60]. An American study examining milk and urine samples of lactating women for glyphosate and aminomethylphosphonic acid (AMPA) did not find any evidence of these chemicals in the breast milk of conventional or organic food consumers [66].

Similar to the findings in urinary output of pesticides found in clinical trial research, cross-sectional analysis of organophosphorus metabolites in children $(n=39)$ show that those consuming organic foods have considerably lower levels of dimethyl metabolites in their urine than those consuming conventional diets $(0.03$ and $0.17 \mu \mathrm{mol} / \mathrm{L}, p<0.001)$, respectively [33].

\subsubsection{Adult Research}

The Nutri-Net Santé Cohort has analysed data from 62,224 participants enrolled in France, through an internet-based survey, with information on frequency of organic food consumption and repeated anthropometric data. The data was predominantly self-reported. An increase in the organic score was associated with a lower risk of being overweight $(\mathrm{OR}=0.77,95 \% \mathrm{CI} 0.68,0.86, p<0.0001)$. The association remained strong and highly significant, with a reduction in the risk of obesity of $37 \%$ after a 3.1-year follow-up [63]. A cross-section of the cohort $(n=8174)$ examined for metabolic syndrome also detailed positive impact of an organic diet with an adjusted prevalence ratio of 0.69 (95\%CI 0.61, 0.78) when comparing the third tertile of organic food in the diet with the first one $(p<0.0001)$ [64]. Additionally, a nested case-control study $(n=300)$ evaluated pesticide metabolites excreted in the urine within the group, finding significantly lower levels of pesticide metabolites among organic consumers versus conventional consumers, with median concentration levels of investigated metabolites for diethylphosphate ( 0.196 versus 0.297$)$, dimethylphosphate $(0.620$ versus 1.382$)$, and total dialkylphosphates $(0.12$ versus 0.16$), p<0.05$ [54]. 
A separate prospective cohort study in adults that estimated organophosphate exposure from food frequency records of 4466 multi-ethnic older Americans, measured urinary pesticide excretion in a sub-group $(n=240)$ and found that higher levels of estimated dietary organophosphate exposure were associated with higher dialkylphosphate concentrations excreted in the urine $(p<0.05)$ [32].

The Million Women Study in the United Kingdom examined any association with cancer incidence and organic diet over a 9-year follow-up period in 1.3 million women. They found no association for reduced cancer incidence in the group, with the exception of a possibly lower incidence of non-Hodgkin lymphoma [65].

The Nutri-Net Santé group also investigated associations with cancer incidence in a cohort of 68,946 participants [53]. The group, followed for a mean of 4.6 years, report that after adjustment for confounders, high organic food scores were linearly and negatively associated with the overall risk of cancer (HR for Q4 vs Q1, 0.75; 95\%CI, 0.63-0.88; $\mathrm{p}$ for trend $=0.001$; absolute risk reduction, $0.6 \%$; HR for a 5-point increase, $0.92 ; 95 \% \mathrm{CI} 0.88-0.96)$. Amongst specific cancers, they found a decreased risk of developing non-Hodgkin lymphoma $(p=0.049)$ and postmenopausal breast cancer, with no association for other types of cancer. The information on non-Hodgkin lymphoma is similar to that found in the Million Women study; however, the information related to breast cancer was in direct contrast.

A nested matched case-control study of 300 participants (150 low and 150 high organic food consumers) within the Nutri-Net Santé had serum samples analysed for differences in nutritional biomarkers [20]. No significant differences were found between the 2 groups for $\alpha$-tocopherol and retinol, cadmium, copper, ferritin or transferrin. Organic consumers exhibited higher plasma concentrations of $\alpha$-carotene, $\beta$-carotene, lutein, and zeaxanthin, whereas no differences were found for other carotenoids ( $\beta$-cryptoxanthin and lycopene). Organic consumers had higher levels of magnesium and a lower plasma concentration of iron. Within the fatty acid analysis, organic consumers had lower palmitoleic acid, $\gamma$-linolenic acid, and docosapentaenoic acid and higher linoleic acid concentrations. The results of these participants, matched for dietary patterns and other health factors, indicates a possible mild modulation of nutritional levels between organic and non-organic consumers.

\subsection{Bias Assessments}

The results of bias assessment for cohort studies showed all studies as good or fair, with no studies returning an assessment of poor. Cross-sectional studies were assessed as having a low risk of bias, with the exception of Jensen et al. (1996), which was a short report, with high bias due to missing detail. Within the clinical trials reviewed, the risk of bias was classified as high in several areas, specifically those related to blinding and allocation concealment. Due to the nature of the intervention, in some cases, it was difficult to adequately blind participants (i.e., food packaging, replacement of 'usual' diet products). There were, however, several studies [37-41] where blinding and randomisation is stated, but the method is not adequately reported and, therefore, they have received an unclear risk of bias in these areas. Many of the studies were not randomised, providing one diet followed by the alternate diet for all participants concurrently.

Significant bias likely to affect the outcomes of the reports was found for two studies conducted by the same research group in Italy $[45,46]$. In both cases, all participants received a controlled Mediterranean diet (MD) for 14 days followed by the same diet for a further 14 days using organic rather than conventional foodstuffs, with no washout between diet arms. This introduces a significant risk of bias for the validity of the outcomes for the organic diet intervention as it may be a cumulative effect of the MD changes, rather than a specific effect for the organic component of the diet.

Another study with high risk of bias was the study by Goen et al. [49] as it contained only two people in the treatment group, in an open-label crossover trial, with no washout between diets. Results of bias assessments are shown in Supplementary Figure S2. 


\subsection{Quality of included Reviews}

No formal grading system was applied to the included articles; however, elements of study quality, including high risk of bias or un-realistic results have been discussed for individual articles throughout the review. Several included articles in this present review were not accepted in the previous systematic review into this topic conducted by Dangour et al. (2010). These include pesticide excretion studies [33,72] and a cross-sectional study on semen analyses [51], excluded on the basis of being contaminant studies; and a second semen analysis study [73], excluded as an occupational health study. The rationale for our inclusion of these studies is that although occupational exposure may have been a factor in the Larsen study [73], the method of calculating pesticide exposure was based entirely on food intake. Pesticide excretion studies were included as this was considered potentially important for health, and these studies are also included in other reviews discussing comparison of organic and conventional food intakes on health, i.e., Smith-Spangler et al. [19].

\section{Discussion}

This systematic review reports on a wide range of interventional (15 publications) and observational studies (20 publications/13 cohorts), where the health effects of organic diet consumption (whole diet or partial replacement) are compared to conventional diet consumption. Substantially more papers are included compared to previous systematic reviews on this topic $[19,35]$ with varying levels of bias and quality.

\subsection{Clinical Trials}

The included clinical trials use a diverse range of methodologies, all involving short-term food substitutions. These range from acute intake of a single dietary item (conventional or organic), to entire diet substitution over a maximum exposure time of 4 weeks, with most of the studies utilising a 2-week intervention period. The majority of the results show no, or minimal, significant differences between organic $(\mathrm{O})$ and non-organic (NO) treatments in the biomarkers selected. In several of these trials, a single food or drink [37-40,42,47,48] was substituted for their organic equivalent. Those studies that also compared the composition of the two food items found there was no difference in the concentration of the nutrient of interest (i.e., lycopene) between $\mathrm{O}$ and $\mathrm{NO}$ foods [37,38,47]. It seems logical, therefore, that a change in participants' samples would seem unlikely unless there was positive laboratory evidence to demonstrate a specific difference between the NO and $\mathrm{O}$ substance that could lead to a biologically plausible difference in vivo.

Similarly, in whole-diet substitution studies, those that examined antioxidant capacity or nutrients in biomarkers, generally did not show between-group differences, which again appeared to be reflective of the laboratory values of these nutrients were measured [41,47]. However, one study did show a significant change in antioxidant capacity [45]. This study, and a related trial [46], which was the only trial to assess a direct health outcome, both provided a NO Mediterranean diet intervention for 2 weeks prior to 2 weeks of the same O Mediterranean diet. There are several issues with the methodology of this model, these and the associated high risk of bias are discussed further in Section 3.5. The reported weight loss and body composition changes in this study appear unrealistic for the 14-day time frame. The authors report a mean weight loss of $5.6 \mathrm{~kg}$, with mean (SD) weight change from the end of NO diet to end of $\mathrm{O}$ diet was $85.17( \pm 13.97)$ to $79.52( \pm 10.41), p=0.0365$. The fat loss is reported as $7.18 \mathrm{~kg}$ over the two week period from $23.36( \pm 8.88)$ to $16.18( \pm 3.34), p=0.0054$, there was also a non-significant $1.18 \mathrm{~kg}$ rise in lean muscle mass, from $53.45( \pm 6.69)$ to $54.63( \pm 6.76)$ [46]. Without baseline assessments provided before any dietary intervention in this group, the effect of the organic intervention cannot be relied upon.

Whole-diet substitution trials that measured changes in pesticide excretion showed significant and substantial reductions during the $\mathrm{O}$ diet phase $[31,34,43,44,49]$, and are discussed under Section 4.3. 
To date, there are no long-term clinical trials measuring direct health outcomes from organic diet intervention. The short timeframe of currently available clinical trials is a serious limitation in assessing demonstrable health benefits. Additionally, only surrogate markers of health have been applied to the majority of clinical trials, with most trials measuring antioxidant levels or pesticide metabolite excretion.

\subsection{Observational Research}

Observational research, which has followed cohorts for up to 10 years (Nutri-Net Santé and the Million Women study), has investigated a range of hypotheses regarding organic diet and health. Studies included in this review report positive associations between organic diet consumption and a range of areas, including fertility, birth defects, allergic sensitisation, non-Hodgkin lymphoma and metabolic syndrome.

Findings from two cross-sectional reports on semen parameters detailed mixed findings, and although the majority of tested parameters showed no significant differences, higher sperm concentration in O consumers [50] and lower normal sperm in NO consumers [51] offer preliminary data that is worthy of further exploration. In female fertility, very positive associations between low dietary pesticide exposure and successful pregnancy and birth outcomes in women undergoing assisted reproduction have been reported in one study [52]. Given the declining fertility rates and poorer semen quality being reported worldwide [74], higher odds of achieving clinical pregnancy and live birth with an organic diet is a significant and important finding. A reduction in risk of birth defects (hypospadias) [55,57], but not cryptorchidism [55], and reduced risk of pre-eclampsia [56] add further evidence for organic diet use through pregnancy.

In children, increased risk of recurrent otitis media has been positively associated with pesticide intake [62], and decreased allergic sensitisation was shown in families following an anthroposophical lifestyle, in comparison to a conventional cohort in the Assessment of Lifestyle and Allergic Disease During Infancy (ALLADIN) study [61]. Consumption of organic dairy products was associated with lower eczema risk as the only significant positive outcome in a similar study (KOALA) [60,70,71]. There are other studies that have supported lower rates of allergic sensitisation from an anthroposophical lifestyle; however, the contribution of organic foods in these studies was not sufficient for them to be included in this review [75-77]. Specific confounding factors related to anthroposophic studies are discussed in Section 4.4 .

The largest studies reporting on adult populations include the Nutri-Net Santé Cohort Study (France), and the Million Women Study (UK). Both of these studies have investigated associations with cancer risk $[64,65]$, with both finding reduced risk of developing non-Hodgkin lymphoma with increased organic consumption. Other findings between the two studies were similar, with a very small risk reduction $(0.6 \%)$ for all cancers in France, but no risk reduction in the UK. Postmenopausal breast cancer rates were decreased in high-O consumers [64], but overall breast cancer risk slightly increased in the alternate study [65]. Different adjustment variables between the studies may have been partly responsible for the different outcomes reported, i.e., the Million Women Study adjusted for hormone replacement in breast cancer, which the Nutri-Net Santé study did not report.

Other findings from the Nutri-Net Santé study show reductions in overweight and risk of obesity, as well as reduced incidence of metabolic syndrome demonstrated in favor of organic food intake $[63,64]$. Whilst this was self-reported data, there is evidence from other association studies that supports dysregulation of several key facets involved in metabolic syndrome in association with serum pesticides [78,79].

As with any observational studies, there is difficulty in determining the causality of the associations that have been observed. It is possible that the benefits of organic diets are associated only with long-term consumption, or result from lifestyle factors or dietary patterns, which is much harder to model in prospective clinical trials. 


\subsection{Pesticide Excretion}

One of the major benefits proposed for organic food is the reduction in exposure to chemicals such as pesticides. Pesticide residues are found in differing amounts across predominantly, fruits and vegetables, but also, grain and dairy products, with much lower amounts found in animal products (except liver, which contains high levels) [24].

The major class of pesticides tested for in the organic food literature reviewed for this paper were the organophosphates, the metabolites of which can be measured in the urine as markers of recent exposure. The most commonly detected metabolites are dimethylphosphate, dimethylthiophosphate, diethylphosphate, and diethylthiophosphate. In some studies, herbicide exposure was also assessed, mainly glyphosate, often assessed through its metabolite aminomethylphosphonic acid. Interventions with organic diets markedly reduced the levels of these compounds, and observational studies in adults and children also show reduced urinary metabolite levels in organic versus conventional diets.

Given that several organophosphorus (OP) insecticides and glyphosate (an OP herbicide and the world's most widely used agricultural chemical) were recently re-classified by the WHO's International Agency for Research on Cancer (IARC) as being "probably carcinogenic" [80], reduced exposure may potentially benefit health. Results of recent reviews comparing pesticide residues in organic and conventional foods conclude that organic food consumption is one approach to substantially minimise exposure to pesticides $[17,21]$.

The impact of switching to organic food consumption on reducing dietary pesticide exposure may be higher in consumers that follow current dietary guidelines for wholegrain and fruit/vegetable consumption. Foods may also be 'pesticide-free' but not 'organic'. It is well documented that pesticide concentrations in wholegrain and wholemeal products are higher than in polished grains such as white flour products (since the outer bran layers of grains have higher pesticide loads then the endosperm) [81]. Apart from wholegrain products, fruits and vegetables are the main dietary source for pesticide exposure and recent European monitoring showed that multiple residues and concentrations above the MRL are most frequently found in fruit and vegetables [24].

\subsection{Confounders of Results}

Lifestyle factors amongst organic consumers are likely to have an important impact on external validity. Organic consumers tend to be more health conscious, are more likely to be vegetarian or vegan and are more likely to be physically active $[7,8]$.

Epidemiological research has shown consumers of organic food generally have a diet that is higher in plant-based food, lower in animal products, with a higher intake of legumes, nuts, and wholegrains than their conventional food-consuming counterparts. These dietary patterns are likely to have significant health benefits in comparison to what is commonly recognised as the standard Western diet, a diet categorised by highly refined, low-fibre, omnivorous diets low in fruits, vegetables and other plant-based foods [82]. A wholefood diet (high in fibre and plant matter) also has demonstrable effects on a healthy diverse microbiota, which is linked to overall health [83]. The organic consumer group may, therefore, not be representative of the general population, i.e., any benefits from organic food consumption may be attributable partly to increased wholefood intake and a healthier lifestyle.

Whole diet composition and diet quality have been measured and adjusted for in different ways in observational research, with varying elements of the diet included as part of the 'organic intake' data collected. It is possible that the benefit observed for organic intake may be partly due to the quality and composition of the diet rather than a direct effect of organic food consumption. Additionally, validation of self-reported organic intake in observational studies is lacking.

The included cohorts from anthroposophical backgrounds (ALLADIN and KOALA birth cohorts) adds an additional layer of confounding, as the consumption of organic food forms only a small part of the dietary measures adopted in this group. Anthroposophy includes a strong focus on fermented foods, biodynamic production, use of butter and olive oil as predominant fats, and long-term breastfeeding $[60,61]$. This is combined with other factors such as reduced levels of antibiotic and 
medication use and a high proportion of plant foods, which together may impact on the overall health of mothers and babies, and influence the results shown.

\subsection{Limitations}

In the included studies, there was wide heterogeneity in the definition and application of the term 'organic' and the percentage of organic food replacement in the diet. This makes any interpretation on the benefits or otherwise of organic food consumption very difficult. No formal grading system was applied to the included studies. A grading criteria, such as that employed by Dangour et al. (2010), would have been helpful to categorise the research according to quality. The review was limited by the non-inclusion of foreign language databases.

\section{Conclusions}

A growing number of important findings are being reported from observational research linking demonstrable health benefits to levels of organic food consumption. Clinical trial research has been short-term and measured largely surrogate markers with limited positive results.

Pesticide excretion studies have consistently shown a reduction in urinary pesticide metabolites with an organic diet; however, there is insufficient evidence to show translation into clinically relevant and meaningful health outcomes. There is a need for studies to move beyond simply measuring the reduction in pesticide exposure with organic food, to investigating measurable health benefits.

The finding that organic food consumption substantially reduces urinary OP levels is important information for consumers, who would like to take a precautionary approach and minimise OP-pesticide exposure. Given the current knowledge on the toxicity of these chemicals, it seems possible that ongoing reduced exposure may translate to health benefits.

While findings from this systematic review showed significant positive outcomes from observational studies in several areas, including reduced incidence of metabolic syndrome, high BMI, non-Hodgkin lymphoma, infertility, birth defects, allergic sensitisation, otitis media and pre-eclampsia, the current evidence base does not allow a definitive statement on the long-term health benefits of organic dietary intake. Consumption of organic food is often tied to overall healthier dietary practices and lower levels of overweight and obesity, which are likely to be influential in the results of observational research.

\section{Recommendations for Future Research}

Single-food substitution studies have shown no benefits and should not be undertaken without substantive pre-clinical data. Additionally, surrogate markers, i.e., antioxidant levels and pesticide excretion, are insufficient to determine actual benefit to health and ideally should be coupled with measurements related to specific health outcomes. Unlike the current exposure studies which measure changes in days or weeks, longer-term health benefit studies are needed. Specifically, long-term whole-diet substitution studies, using certified organic interventions will provide the most reliable evidence to answer the question of whether an organic diet provides true measurable health benefits.

Additional research options may include further evaluation of biological data collected through previous large cohort studies, such as the Nutri-Net Santé study [84], and the MoBa biobank [85], to test hypotheses on organic diet and health.

Supplementary Materials: The following are available online at http://www.mdpi.com/2072-6643/12/1/7/s1, Figure S1: Medline search strategy, Figure S2: Risk of bias summary tables.

Author Contributions: Conceptualization, S.M. and C.L.; methodology, S.M. and V.V.; data curation, V.V.; writing—original draft preparation, V.V.; S.M.; C.O.; J.A.; S.R.; writing—review and editing, V.V.; C.O. All authors have read and agreed to the published version of the manuscript.

Funding: A grant from the Pro Vice-Chancellor (Research) at Southern Cross University partially funded this study. 
Conflicts of Interest: The authors declare no conflict of interest. The research team are associated with a research centre in organic food, and have remained mindful to ensure this review was objective, transparent and reproducible.

\section{References}

1. IFOAM. Consolidated Annual Report of IFOAM-Organics International; IFOAM: Bonn, Germany, 2018.

2. Willer, H.; Lernoud, J. The World of Organic Agriculture. Statistics and Emerging Trends; Research Institute of Organic Agriculture (FiBL) \& IFOAM-Organics International: Frick, Switzerland, 2017.

3. USDA. U.S. Organic Food Sales by Category: 2005-14E. Available online: https://www.ers.usda.gov/topics/ natural-resources-environment/organic-agriculture/organic-market-overview/ (accessed on 31 July 2018).

4. Apaolaza, V.; Hartmann, P.; D’Souza, C.; López, C.M. Eat organic-Feel good? The relationship between organic food consumption, health concern and subjective wellbeing. Food Qual. Prefer. 2018, 63, 51-62. [CrossRef]

5. Hoefkens, C.; Verbeke, W.; Aertsens, J.; Mondelaers, K.; Van Camp, J. The nutritional and toxicological value of organic vegetables. Br. Food. J. 2009, 111, 1062-1077. [CrossRef]

6. van de Vijver, L.P.L.; van Vliet, M.E.T. Health effects of an organic diet-Consumer experiences in the Netherlands. J. Sci. Food Agric. 2012, 92, 2923-2927. [CrossRef] [PubMed]

7. Baudry, J.; Méjean, C.; Péneau, S.; Galan, P.; Hercberg, S.; Lairon, D.; Kesse-Guyot, E. Health and dietary traits of organic food consumers: Results from the NutriNet-Santé study. Br. J. Nutr. 2015, 114, 2064-2073. [CrossRef]

8. Eisinger-Watzl, M.; Wittig, F.; Heuer, T.; Hoffmann, I. Customers purchasing organic food-Do they live healthier? Results of the German National Nutrition Survey II. Eur. J. Nutr. Food Saf. 2015, 5, 59-71. [CrossRef]

9. Simões-Wüst, A.P.; Moltó-Puigmartí, C.; van Dongen, M.C.; Dagnelie, P.C.; Thijs, C. Organic food consumption during pregnancy is associated with different consumer profiles, food patterns and intake: The KOALA Birth Cohort Study. Public Health Nutr. 2017, 20, 2134-2144. [CrossRef]

10. Barański, M.; Średnicka-Tober, D.; Volakakis, N.; Seal, C.; Sanderson, R.; Stewart, G.B.; Benbrook, C.; Biavati, B.; Markellou, E.; Giotis, C.; et al. Higher antioxidant and lower cadmium concentrations and lower incidence of pesticide residues in organically grown crops: A systematic literature review and meta-analyses. Br. J. Nutr. 2014, 112, 794-811. [CrossRef]

11. Benbrook, C.M.; Butler, G.; Latif, M.A.; Leifert, C.; Davis, D.R. Organic Production Enhances Milk Nutritional Quality by Shifting Fatty Acid Composition: A United States-Wide, 18-Month Study. PLoS ONE 2013, 8, e82429. [CrossRef]

12. Palupi, E.; Jayanegara, A.; Ploeger, A.; Kahl, J. Comparison of nutritional quality between conventional and organic dairy products: A meta-analysis. J. Sci. Food Agric. 2012, 92, 2774-2781. [CrossRef]

13. Srednicka-Tober, D.; Baranski, M.; Seal, C.; Sanderson, R.; Benbrook, C.; Steinshamn, H.; Gromadzka-Ostrowska, J.; Rembialkowska, E.; Skwarlo-Sonìta, K.; Eyre, M.; et al. Higher PUFA and n-3 PUFA, conjugated linoleic acid, [...]-tocopherol and iron, but lower iodine and selenium concentrations in organic milk: A systematic literature review and meta- and redundancy analyses. Br. J. Nutr. 2016, 115, 1043-1060. [CrossRef]

14. Ribas-Agusti, A.; Diaz, I.; Sarraga, C.; Garcia-Regueiro, J.A.; Castellari, M. Nutritional properties of organic and conventional beef meat at retail. J. Sci. Food Agric. 2019, 99, 4218-4225. [CrossRef] [PubMed]

15. Srednicka-Tober, D.; Baranski, M.; Seal, C.; Sanderson, R.; Benbrook, C.; Steinshamn, H.; Gromadzka-Ostrowska, J.; Rembialkowska, E.; Skwarlo-Sonìta, K.; Eyre, M.; et al. Composition differences between organic and conventional meat: A systematic literature review and meta-analysis. Br. J. Nutr. 2016, 115, 994-1011. [CrossRef] [PubMed]

16. Barański, M.; Rempelos, L.; Iversen, P.O.; Leifert, C. Effects of organic food consumption on human health; the jury is still out! Food Nutr. Res. 2017, 61, 1287333. [CrossRef]

17. Brantsæter, A.L.; Ydersbond, T.A.; Hoppin, J.A.; Haugen, M.; Meltzer, H.M. Organic food in the diet: Exposure and health implications. Annu. Rev. Public Health 2017, 38, 295-313. [CrossRef] [PubMed]

18. Hurtado-Barroso, S.; Tresserra-Rimbau, A.; Vallverdu-Queralt, A.; Lamuela-Raventos, R.M. Organic food and the impact on human health. Crit. Rev. Food Sci. Nutr. 2017, 59, 704-714. [CrossRef] [PubMed] 
19. Smith-Spangler, C.; Brandeau, M.L.; Hunter, G.E.; Clay Bavinger, J.; Pearson, M.; Eschbach, P.J.; Sundaram, V.; Liu, H.; Schirmer, P.; Stave, C.; et al. Are organic foods safer or healthier than conventional alternatives?: A systematic review. Ann. Intern. Med. 2012, 157, 348-366. [CrossRef]

20. Baudry, J.; Ducros, V.; Druesne-Pecollo, N.; Galan, P.; Hercberg, S.; Debrauwer, L.; Amiot, M.J.; Lairon, D.; Kesse-Guyot, E. Some Differences in Nutritional Biomarkers are Detected Between Consumers and Nonconsumers of Organic Foods: Findings from the BioNutriNet Project. Curr. Dev. Nutr. 2018, 3, nzy090. [CrossRef]

21. Mie, A.; Andersen, H.R.; Gunnarsson, S.; Kahl, J.; Kesse-Guyot, E.; Rembiałkowska, E.; Quaglio, G.; Grandjean, P. Human health implications of organic food and organic agriculture: A comprehensive review. Environ. Health 2017, 16, 111. [CrossRef]

22. European Environment Agency. Late Lessons from Early Warnings: Science, Precaution, Innovation; European Environment Agency: Copenhagen, Danmark, 2013.

23. Mesnage, R.; Antoniou, M.N. Ignoring adjuvant toxicity falsifies the safety profile of commercial pesticides. Front. Public Health 2018, 5, 361. [CrossRef]

24. EFSA. The 2016 European Union report on pesticide residues in food. EFSA J. 2018, 16, 5348.

25. United States Department of Agriculture. Pesticide Data Program: Annual Summary; United States Department of Agriculture: Washington, DC, USA, 2017; pp. 1-203.

26. Lydy, M.; Belden, J.; Wheelock, C.; Hammock, B.; Denton, D. Challenges in regulating pesticide mixtures. Ecol. Soc. 2004, 9, 1. [CrossRef]

27. Rizzati, V.; Briand, O.; Guillou, H.; Gamet-Payrastre, L. Effects of pesticide mixtures in human and animal models: An update of the recent literature. Chem. Biol. Interact. 2016, 254, 231-246. [CrossRef]

28. Mostafalou, S.; Abdollahi, M. Pesticides: An update of human exposure and toxicity. Arch. Toxicol. 2017, 91, 549-599. [CrossRef] [PubMed]

29. Tago, D.; Andersson, H.; Treich, N. Pesticides and health: A review of evidence on health effects, valuation of risks, and benefit-cost analysis. Adv. Health Econ. Health Serv. Res. 2014, 24, 203-295. [PubMed]

30. WHO. Pesticide Residues in Food-2016: Toxicological Evaluations. In Proceedings of the Joint Meeting of the FAO Panel of Experts on Pesticide Residues in Food and the Environment and the WHO Core Assessment Group on Pesticide Residues, Geneva, Switzerland, 9-13 May 2016.

31. Bradman, A.; Quirós-Alcalá, L.; Castorina, R.; Schall, R.A.; Camacho, J.; Holland, N.T.; Barr, D.B.; Eskenazi, B. Effect of organic diet intervention on pesticide exposures in young children living in low-income urban and agricultural communities. Environ. Health Perspect. 2015, 123, 1086-1093. [CrossRef] [PubMed]

32. Curl, C.L.; Beresford, S.A.A.; Fenske, R.A.; Fitzpatrick, A.L.; Lu, C.; Nettleton, J.A.; Kaufman, J.D. Estimating pesticide exposure from dietary intake and organic food choices: The Multi-Ethnic Study of Atherosclerosis (MESA). Environ. Health Perspect. 2015, 123, 475-483. [CrossRef] [PubMed]

33. Curl, C.L.; Fenske, R.A.; Elgethum, K. Organophosphorus pesticide exposure of urban and suburban preschool children with organic and conventional diets. Environ. Health Perspect. 2003, 111, 377-382. [CrossRef]

34. Oates, L.; Cohen, M.; Braun, L.; Schembri, A.; Taskova, R. Reduction in urinary organophosphate pesticide metabolites in adults after a week-long organic diet. Environ. Res. 2014, 132, 105-111. [CrossRef]

35. Dangour, A.D.; Lock, K.; Hayter, A.; Aikenhead, A.; Allen, E.; Uauy, R. Nutrition-related health effects of organic foods: A systematic review. Am. J. Clin. Nutr. 2010, 92, 203-210. [CrossRef]

36. Moher, D.; Liberati, A.; Tetzlaff, J.; Altman, D.G.; Group, T.P. Preferred Reporting Items for Systematic Reviews and Meta-Analyses: The PRISMA Statement. Ann. Intern. Med. 2009, 151, 264-269. [CrossRef]

37. Caris-Veyrat, C.; Amiot, M.J.; Tyssandier, V.; Grasselly, D.; Buret, M.; Mikolajczak, M.; Guilland, J.C.; Bouteloup-Demange, C.; Borel, P. Influence of organic versus conventional agricultural practice on the antioxidant microconstituent content of tomatoes and derived purees; consequences on antioxidant plasma status in humans. J. Agric. Food Chem. 2004, 52, 6503-6509. [CrossRef] [PubMed]

38. Stracke, B.A.; Rufer, C.E.; Bub, A.; Briviba, K.; Seifert, S.; Kunz, C.; Watzl, B. Bioavailability and nutritional effects of carotenoids from organically and conventionally produced carrots in healthy men. Br. J. Nutr. 2009, 101, 1664-1672. [CrossRef] [PubMed]

39. Stracke, B.A.; Rufer, C.E.; Bub, A.; Seifert, S.; Weibel, F.P.; Kunz, C.; Watzl, B. No effect of the farming system (organic/conventional) on the bioavailability of apple (Malus domestica Bork, cultivar Golden Delicious) polyphenols in healthy men: A comparative study. Eur. J. Nutr. 2010, 49, 301-310. [CrossRef] [PubMed] 
40. Briviba, K.; Stracke, B.A.; Rufer, C.E.; Watzl, B.; Weibel, F.P.; Bub, A. Effect of consumption of organically and conventionally produced apples on antioxidant activity and DNA damage in humans. J. Agric. Food Chem. 2007, 55, 7716-7721. [CrossRef] [PubMed]

41. Grinder-Pedersen, L.; Rasmussen, S.E.; Bugel, S.; Jorgensen, L.V.; Dragsted, L.O.; Gundersen, V.; Sandstrom, B. Effect of diets based on foods from conventional versus organic production on intake and excretion of flavonoids and markers of antioxidative defense in humans. J. Agric. Food Chem. 2003, 51, 5671-5676. [CrossRef] [PubMed]

42. Akçay, Y.D.; Yıldırım, H.K.; Güvenç, U.; Sözmen, E.Y. The effects of consumption of organic and nonorganic red wine on low-density lipoprotein oxidation and antioxidant capacity in humans. Nutr. Res. 2004, 24, 541-554. [CrossRef]

43. Lu, C.; Toepel, K.; Irish, R.; Fenske, R.A.; Barr, D.B.; Bravo, R. Organic diets significantly lower children's dietary exposure to organophosphorus pesticides. Environ. Health Perspect. 2006, 114, 260-263. [CrossRef]

44. Lu, C.; Barr, D.B.; Pearson, M.A.; Waller, L.A. Dietary intake and its contribution to longitudinal organophosphorus pesticide exposure in urban/suburban children. Environ. Health Perspect. 2008, 116, 537-542. [CrossRef]

45. Di Renzo, L.; Di Pierro, D.; Bigioni, M.; Sodi, V.; Galvano, F.; Cianci, R.; La Fauci, L.; De Lorenzo, A. Is antioxidant plasma status in humans a consequence of the antioxidant food content influence? Eur. Rev. Med. Pharmacol. Sci. 2007, 11, 185-192.

46. De Lorenzo, A.; Noce, A.; Bigioni, M.; Calabrese, V.; Della Rocca, D.G.; Di Daniele, N.; Tozzo, C.; Di Renzo, L. The effects of Italian Mediterranean organic diet (IMOD) on health status. Curr. Pharm. Des. 2010, 16, 814-824. [CrossRef]

47. Søltoft, M.; Bysted, A.; Madsen, K.H.; Mark, A.B.; Bügel, S.G.; Nielsen, J.; Knuthsen, P. Effects of organic and conventional growth systems on the content of carotenoids in carrot roots, and on intake and plasma status of carotenoids in humans. J. Sci. Food Agric. 2011, 91, 767-775. [CrossRef] [PubMed]

48. Toaldo, I.M.; Cruz, F.A.; da Silva, E.L.; Bordignon-Luiz, M.T. Acute consumption of organic and conventional tropical grape juices (Vitis labrusca L.) increases antioxidants in plasma and erythrocytes, but not glucose and uric acid levels, in healthy individuals. Nutr. Res. 2016, 36, 808-817. [CrossRef] [PubMed]

49. Goen, T.; Schmidt, L.; Lichtensteiger, W.; Schlumpf, M. Efficiency control of dietary pesticide intake reduction by human biomonitoring. Int. J. Hyg. Environ. Health 2017, 220, 254-260. [CrossRef] [PubMed]

50. Jensen, T.K.; Giwercman, A.; Carlsen, E.; Scheike, T.; Skakkebaek, N.E. Semen quality among members of organic food associations in Zealand, Denmark. Lancet 1996, 347, 1844. [CrossRef]

51. Juhler, R.K.; Larsen, S.B.; Meyer, O.; Jensen, N.D.; Spano, M.; Giwercman, A.; Bonde, J.P. Human semen quality in relation to dietary pesticide exposure and organic diet. Arch. Environ. Contam. Toxicol. 1999, 37, 415-423. [CrossRef]

52. Chiu, Y.H.; Williams, P.L.; Gillman, M.W.; Gaskins, A.J.; Minguez-Alarcon, L.; Souter, I.; Toth, T.L.; Ford, J.B.; Hauser, R.; Chavarro, J.E.; et al. Association between pesticide residue intake from consumption of fruits and vegetables and pregnancy outcomes among women undergoing infertility treatment with assisted reproductive technology. JAMA Intern. Med. 2018, 178, 17-26. [CrossRef]

53. Baudry, J.; Assmann, K.E.; Touvier, M.; Allès, B.; Seconda, L.; Latino-Martel, P.; Ezzedine, K.; Galan, P.; Hercberg, S.; Lairon, D.; et al. Association of frequency of organic food consumption with cancer risk: Findings from the nutrinet-santé prospective cohort study. JAMA Intern. Med. 2018, 178, 1597-1606. [CrossRef]

54. Baudry, J.; Debrauwer, L.; Durand, G.; Limon, G.; Delcambre, A.; Vidal, R.; Taupier-Letage, B.; Druesne-Pecollo, N.; Galan, P.; Hercberg, S.; et al. Urinary pesticide concentrations in French adults with low and high organic food consumption: Results from the general population-based NutriNet-Santé. J. Expo. Sci. Environ. Epidemiol. 2019, 29, 366-378. [CrossRef]

55. Brantsæter, A.L.; Torjusen, H.; Meltzer, H.M.; Papadopoulou, E.; Hoppin, J.A.; Alexander, J.; Lieblein, G.; Roos, G.; Holten, J.M.; Swartz, J.; et al. Organic food consumption during pregnancy and hypospadias and cryptorchidism at birth: The Norwegian Mother and Child Cohort Study (MoBa). Environ. Health Perspect. 2016, 124, 357-364. [CrossRef]

56. Torjusen, H.; Brantsæter, A.L.; Haugen, M.; Alexander, J.; Bakketeig, L.S.; Lieblein, G.; Stigum, H.; Næs, T.; Swartz, J.; Holmboe-Ottesen, G.; et al. Reduced risk of pre-eclampsia with organic vegetable consumption: Results from the prospective Norwegian Mother and Child Cohort Study. BMJ Open 2014, 4, 6143. [CrossRef] 
57. Christensen, J.S.; Asklund, C.; Skakkebaek, N.E.; Jorgensen, N.; Andersen, H.R.; Jorgensen, T.M.; Olsen, L.H.; Hoyer, A.P.; Moesgaard, J.; Thorup, J.; et al. Association between organic dietary choice during pregnancy and hypospadias in offspring: A study of mothers of 306 boys operated on for hypospadias. J. Urol. 2013, 189, 1077-1082. [CrossRef] [PubMed]

58. Rist, L.; Mueller, A.; Barthel, C.; Snijders, B.; Jansen, M.; Simões-Wüst, A.P.; Huber, M.; Kummeling, I.; von Mandach, U.; Steinhart, H.; et al. Influence of organic diet on the amount of conjugated linoleic acids in breast milk of lactating women in the Netherlands. Br. J. Nutr. 2007, 97, 735-743. [CrossRef] [PubMed]

59. Mueller, A.; Steinhart, H.; Thijs, C.; Rist, L.; Simoes-Wust, A.P.; Huber, M. Trans fatty acids in human milk are an indicator of different maternal dietary sources containing trans fatty acids. Lipids 2010, 45, 245-251. [CrossRef] [PubMed]

60. Kummeling, I.; Thijs, C.; Huber, M.; van de Vijver, L.P.L.; Snijders, B.E.P.; Penders, J.; Stelma, F.; van Ree, R.; van den Brandt, P.A.; Dagnelie, P.C. Consumption of organic foods and risk of atopic disease during the first 2 years of life in the Netherlands. Br. J. Nutr. 2008, 99, 598-605. [CrossRef] [PubMed]

61. Stenius, F.; Swartz, J.; Lilja, G.; Borres, M.; Bottai, M.; Pershagen, G.; Scheynius, A.; Alm, J. Lifestyle factors and sensitization in children-the ALADDIN birth cohort. Allergy 2011, 66, 1330-1338. [CrossRef]

62. Buscail, C.; Chevrier, C.; Serrano, T.; Pele, F.; Monfort, C.; Cordier, S.; Viel, J.F. Prenatal pesticide exposure and otitis media during early childhood in the PELAGIE mother-child cohort. Occup. Environ. Med. 2015, 72, 837-844. [CrossRef]

63. Kesse-Guyot, E.; Baudry, J.; Assmann, K.E.; Galan, P.; Hercberg, S.; Lairon, D. Prospective association between consumption frequency of organic food and body weight change, risk of overweight or obesity: Results from the NutriNet-Santé Study. Br. J. Nutr. 2017, 117, 325-334. [CrossRef]

64. Baudry, J.; Lelong, H.; Adriouch, S.; Julia, C.; Allès, B.; Hercberg, S.; Touvier, M.; Lairon, D.; Galan, P.; Kesse-Guyot, E. Association between organic food consumption and metabolic syndrome: Cross-sectional results from the NutriNet-Santé study. Eur. J. Nutr. 2017, 57, 2477-2488. [CrossRef]

65. Bradbury, K.E.; Balkwill, A.; Spencer, E.A.; Roddam, A.W.; Reeves, G.K.; Green, J.; Key, T.J.; Beral, V.; Pirie, K. Organic food consumption and the incidence of cancer in a large prospective study of women in the United Kingdom. Br. J. Cancer 2014, 110, 2321-2326. [CrossRef]

66. McGuire, M.K.; McGuire, M.A.; Price, W.J.; Shafii, B.; Carrothers, J.M.; Lackey, K.A.; Goldstein, D.A.; Jensen, P.K.; Vicini, J.L. Glyphosate and aminomethylphosphonic acid are not detectable in human milk. Am. J. Clin. Nutr. 2016, 103, 1285-1290. [CrossRef]

67. Higgins, J.P.T.; Altman, D.G.; Gøtzsche, P.C.; Jüni, P.; Moher, D.; Oxman, A.D.; Savović, J.; Schulz, K.F.; Weeks, L.; Sterne, J.A.C. The Cochrane Collaboration's tool for assessing risk of bias in randomised trials. BMJ 2011, 343, d5928. [CrossRef] [PubMed]

68. Specialist Unit for Review Evidence (SURE). Questions to Assist with the Critical Appraisal of Cross-Sectional Studies. Available online: https://www.cardiff.ac.uk/specialist-unit-for-review-evidence/resources/criticalappraisal-checklists (accessed on 23 January 2019).

69. Wells, G.; Shea, B.; O'Connell, D.; Peterson, J.; Welch, V.; Losos, M.; Tugwell, P. The Newcastle-Ottawa Scale (NOS) for Assessing the Quality of Nonrandomised Studies in Meta-Analyses. Available online: http://www.ohri.ca/programs/clinical_epidemiology/oxford.asp (accessed on 22 March 2019).

70. Scheepers, L.E.J.M.; Penders, J.; Mbakwa, C.A.; Thijs, C.; Mommers, M.; Arts, I.C.W. The intestinal microbiota composition and weight development in children: The KOALA Birth Cohort Study. Int. J. Obes. 2015, 39, 16-25. [CrossRef] [PubMed]

71. Simoes-Wust, A.P.; Ischa, K.; Monique, M.; Machteld, H.; Lukas, R.; Van De Vijver, L.; Dagnelie, P.C.; Carel, T. Influence of alternative lifestyles on health status and health risk factors in pregnancy and maternity. Eur. J. Integr. Med. 2012, 4, 182-183.

72. Lu, C.; Barr, D.B.; Pearson, M.; Bartell, S.; Bravo, R. A longitudinal approach to assessing urban and suburban children's exposure to pyrethroid pesticides. Environ. Health Perspect. 2006, 114, 1419-1423. [CrossRef] [PubMed]

73. Larsen, S.B.; Spano, M.; Giwercman, A.; Bonde, J.P. Semen quality and sex hormones among organic and traditional Danish farmers. ASCLEPIOS Study Group. Occup. Environ. Med. 1999, 56, 139-144. [CrossRef]

74. Skakkebaek, N.E.; Jørgensen, N.; Andersson, A.-M.; Juul, A.; Main, K.M.; Jensen, T.K.; Toppari, J. Populations, decreasing fertility, and reproductive health. Lancet 2019, 393, 1500-1501. [CrossRef] 
75. Alfven, T.; Braun-Fahrlander, C.; Brunekreef, B.; von Mutius, E.; Riedler, J.; Scheynius, A.; van Hage, M.; Wickman, M.; Benz, M.R.; Budde, J.; et al. Allergic diseases and atopic sensitization in children related to farming and anthroposophic lifestyle: The PARSIFAL study. Allergy 2006, 61, 414-421. [CrossRef]

76. Alm, J.; Swartz, J.; Lilja, G.; Scheynius, A.; Pershagen, G. Atopy in children of families with an anthroposophic lifestyle. Lancet 1999, 353, 1485. [CrossRef]

77. Flöistrup, H.; Swartz, J.; Bergström, A.; Alm, J.S.; Scheynius, A.; van Hage, M.; Waser, M.; Braun-Fahrländer, C.; Schram-Bijkerk, D.; Huber, M.; et al. Allergic disease and sensitization in Steiner school children. J. Allergy Clin. Immunol. 2006, 117, 59-66. [CrossRef]

78. Lee, D.H.; Lee, I.K.; Porta, M.; Steffes, M.; Jacobs, D.R. Relationship between serum concentrations of persistent organic pollutants and the prevalence of metabolic syndrome among non-diabetic adults: Results from the National Health and Nutrition Examination Survey 1999-2002. Diabetologia 2007, 50, 1841-1851. [CrossRef]

79. Lee, D.-H.; Steffes, M.W.; Sjödin, A.; Jones, R.S.; Needham, L.L.; Jacobs, D.R., Jr. Low dose organochlorine pesticides and polychlorinated biphenyls predict obesity, dyslipidemia, and insulin resistance among people free of diabetes. PLoS ONE 2011, 6, e15977. [CrossRef] [PubMed]

80. Guyton, K.Z.; Loomis, D.; Grosse, Y.; El Ghissassi, F.; Benbrahim-Tallaa, L.; Guha, N.; Scoccianti, C.; Mattock, H.; Straif, K. Carcinogenicity of tetrachlorvinphos, parathion, malathion, diazinon, and glyphosate. Lancet Oncol. 2015, 16, 490-491. [CrossRef]

81. Kaushik, G.; Satya, S.; Naik, S.N. Food processing a tool to pesticide residue dissipation-A review. Food Res. Int. 2009, 42, 26-40. [CrossRef]

82. Conlon, M.A.; Bird, A.R. The impact of diet and lifestyle on gut microbiota and human health. Nutrients 2014, 7, 17-44. [CrossRef] [PubMed]

83. De Filippo, C.; Cavalieri, D.; Di Paola, M.; Ramazzotti, M.; Poullet, J.B.; Massart, S.; Collini, S.; Pieraccini, G.; Lionetti, P. Impact of diet in shaping gut microbiota revealed by a comparative study in children from Europe and rural Africa. Proc. Natl. Acad. Sci. USA 2010, 107, 14691-14696. [CrossRef]

84. Hercberg, S.; Castetbon, K.; Czernichow, S.; Malon, A.; Mejean, C.; Kesse, E.; Touvier, M.; Galan, P. The Nutrinet-Santé Study: A web-based prospective study on the relationship between nutrition and health and determinants of dietary patterns and nutritional status. BMC Public Health 2010, 10, 242. [CrossRef]

85. Ronningen, K.S.; Paltiel, L.; Meltzer, H.M.; Nordhagen, R.; Lie, K.K.; Hovengen, R.; Haugen, M.; Nystad, W.; Magnus, P.; Hoppin, J.A. The biobank of the Norwegian Mother and Child Cohort Study: A resource for the next 100 years. Eur. J. Epidemiol. 2006, 21, 619-625. [CrossRef] 